\title{
Quantitative damage and detwinning analysis of nanotwinned copper foil under cyclic loading
}

Byung-Gil Yoo ${ }^{1}$, Steven T. Boles ${ }^{1,2}$, Y. Liu ${ }^{3}$, X. Zhang ${ }^{3,4}$, Ruth Schwaiger ${ }^{1}$, Christoph Eberl ${ }^{5,6}$, Oliver Kraft ${ }^{*}$

1 Institute for Applied Materials, Karlsruhe Institute of Technology, Karlsruhe 76344, Germany

2 Department of Electrical Engineering, The Hong Kong Polytechnic University, Hung Hom, Kowloon, Hong Kong.

3 Department of Materials Science and Engineering, Texas A \& M University, College Station, Texas 77843-3123, USA

4 Department of Mechanical Engineering, Texas A \& M University, College Station, Texas 77843-3123, USA

5 Fraunhofer Institute for Mechanics of Materials (IWM), Freiburg 79108, Germany.

6 Institute for Microsystems Technology, University of Freiburg, Freiburg 79110, Germany.

* Corresponding author.

Electronic address: oliver.kraft@kit.edu

\begin{abstract}
High-purity $\mathrm{Cu}$ samples containing parallel columns of highly aligned nanotwins with median spacing of about $25 \mathrm{~nm}$ were subjected to tension-compression cyclic loading by a highthroughput cyclic testing method. The methodology utilizes gradients in surface strain amplitude of a vibrating cantilever: one along the beam axis, with decreasing strain from the fixed to the free end of the beam, and the other through the foil thickness with decreasing strain from the surface to the neutral axis. Systematic microstructural investigations indicate that nanotwins are not stable under cyclic loading and that the applied strain amplitude has a
\end{abstract}


strong influence on the resulting twin structure. In the highly stressed regions the detwinning process produces a twin free microstructure, allowing for subsequent extrusion and crack formation and introduces fatal defects into structural parts.

Keywords: Fatigue; High-throughput test; Nanotwinned metal; Detwinning; Copper 


\section{Introduction}

Nanotwinned (nt) metals have been studied extensively because of novel mechanical and electrical properties that emerge as the average twin thickness is reduced to nanometer scale [1-2]. It has been shown that nt metals have a strength comparable to that of nanocrystalline (nc) metals, with twin boundary (TB) spacing serving as an analog to that of grain size [1-6]. However, unlike nc metals the significant strength increase in nt metals does not cause a pronounced decrease in ductility. This suggests that the fundamental plastic deformation mechanisms which govern the behavior of nt metals must be different from that in nc metals.

An important advantage of the nt microstructure is its higher stability as compared to nc metals which have been found to undergo extensive and rapid grain growth under stress [714]. Also, the motion of grain boundaries itself has been discussed as a mechanism for plastic deformation [15-18]. Although the grain structure in nt metals has a higher resistance against coarsening compared to nc metals, the mobility of TB can cause detwinning [7-10], leading locally to diminished material properties. Twin stability is highly correlated to the role of TBs and the mechanisms that allow TBs to move, thereby causing twins to grow or shrink (and even disappear) [7-14]. Previous studies have investigated detwinning behavior by in situ testing methods with monotonic loading [19-23] and atomic scale simulations [24-25] with indications that the nano-scale boundary migration and rationalized flow behavior of nt materials are caused by dislocation migration in/near the twin boundary [19-27]. However, only a few studies have been carried out so far on the behavior of nt materials under cyclic loading conditions [27-29].

Recently, a high-throughput fatigue testing technique has been developed for analyzing small-scale phenomena in thin films deposited on Si cantilevers [30]. The technique is based on the vibrating reed test that has been applied, e.g., to multilayered metallic specimens [31]. Due to the stress and strain distribution along the cantilever during testing, 
one can study the influence of strain amplitude on damage formation during cyclic loading based on one test of the same cantilever [30]. In this study, this method has been used for gaining new insights into the detwinning behavior of nt materials under cyclic loading conditions in a systematic and reproducible fashion. In particular, the evolution of nt microstructure as a function of cyclic strain (or stress) amplitude is studied by applying detailed electron microscopy. It will be shown that the stability of the nanostructured material is crucial for the fatigue resistance of this new class of materials.

\section{Experimental}

The fatigue testing methodology of Burger, et al. [30] has been modified to allow for the investigation of microstructure evolution of $\mathrm{nt} \mathrm{Cu}$ under cyclic loading. A schematic of the setup is shown in figure 1. The expected damage propagation along the long axis of the cantilever can be analyzed with increasing number of cycles during testing [30]. This method is complementary to other methods, such as tension-tension and rotating beam methods, as in the high cycle regime the microstructure and damage evolution can be studied simultaneously for different strain amplitudes with less alignment problems. However, it is not well suited to systematically determine lifetimes because only a small volume is stressed at the largest amplitude. This method applies fully reversible compression-tension strain to the specimen (i.e. the strain ratio, $\varepsilon_{\min } / \varepsilon_{\max }, \mathrm{R}_{\varepsilon}=-1$ ) [30-31].

\subsection{Testing setup}

A weight is fixed to the cantilever foil to adjust the resonance frequency. Then the cantilever is shaken by a piezo actuator applying a frequency sweep to find the resonance frequency. For this, the deflection of the cantilever is measured at any position along the cantilever [30-31] by a laser system shown in figure 1 . As an example, the measured deflection at a selected position of the cantilever is plotted versus the frequency in the inset of 
figure 1 . The resonance frequency is obtained precisely from the maximum deflection point. For the cantilever tested here, the FWHM of the resonance peak is smaller than one-hundredth of the resonance frequency and the amplitude drops to less than $5 \%$ of the maximum at $1 \mathrm{~Hz}$ above or below the resonance frequency of roughly $27 \mathrm{~Hz}$. Cyclic-load testing of the cantilever was performed by locking the piezo actuator to the corresponding resonance frequency of the beam. No significant frequency shift due to the damage propagation during cyclic bending was observed. Once cracks develop near the fixed end, the resonance frequency of the sample changes dramatically. After this, there is no vibration under the bending condition. The hardware and data transferred in the setup were controlled by a LabVIEW program (National Instruments, TX, USA).

\subsection{Strain distribution and damage propagation}

Excitation of the cantilever at the resonance frequency of the first bending mode leads to cyclic strains in the self-supported cantilever with the amplitude depending on the position along the cantilever (see figure 2). From beam theory, it is known that the strain decreases from the fixed to the free end of the cantilever. Therefore, the strain amplitude at any position on the surface of the cantilever can be calculated when the maximum deflection of the cantilever is known. Because of the variation of the strain amplitude, the fatigue damage in the cantilever is expected to vary along the cantilever.

The equivalent von Mises strain in the cantilever was calculated by FE simulation (Multiphysics, ANSYS, PA, USA) using a simplified 2D model of the copper cantilever (length $8 \mathrm{~mm}$, thickness $20 \mu \mathrm{m}$ ) [30] and an elastic modulus of $120 \mathrm{GPa}$ for $\mathrm{nt} \mathrm{Cu}$ [2]. The simulation took into account the weight that was attached to the free end of the cantilever. It was adjusted to match the resonance frequency of $\sim 27 \mathrm{~Hz}$ from the experimental observation. Figure 2 shows the strain amplitude values at the surface along the position of the cantilever. As expected, the calculated strain amplitude in the cantilever reaches its maximum at the 
fixed end; it decreases when moving along the cantilever, first in a non-linearly fashion due to the clamping, and then linearly to zero at the free end. Thus by investigating microstructural changes at different positions along the cantilever, information and insight is gained into the damage evolution as a function of strain amplitude when the test is interrupted at different numbers of cycles. Moreover, the applied strain amplitude is largest at the surface of the cantilever and decreases towards the neutral axis in the center through the thickness of the cantilever. Therefore microstructural changes are not only studied at the surface of the beam but also into the depth.

\subsection{Cantilever material}

The $\mathrm{Cu}$ foil with a thickness of $20 \pm 0.2 \mu \mathrm{m}$ was deposited by magnetron sputtering onto single crystal silicon (110) substrates. The thickness was determined by using an ultraviolet laser scanning microscope (UV LSM, KEYENCE, VK-9700K, NJ, USA). The nt microstructure was obtained following the procedure described in [7-8] with an in-between twin thickness of $25 \mathrm{~nm}$. The deposition chamber was evacuated to a base pressure of approximately $8.0 \times 10^{-8}$ Torr and Argon pressure during deposition was $\sim 2$ mTorr. X-ray diffraction (Bruker-AXS D8 Bragg-Brentano X-ray Diffractometer, $\mathrm{Cu}$ Ka radiation) was used to confirm the expected (111) out-of-plane texture in the nt foils [7-8].

The Si substrate was removed from the nt-Cu thin foil using HF etching. Then the foilcantilevers were cut by electro discharge machining to a rectangular shape with a width of 0.8 $\mathrm{mm}$, a length of $8 \mathrm{~mm}$. Then fine milling for edges of the cantilever was performed by a Focused Ion Beam (FIB) milling system (FEI Nova Nanolab 200 dual beam, Netherlands) at $30 \mathrm{kV}$ and a beam current of $20 \mathrm{nA}$. Subsequently, the cantilever was gripped to a custommade Al holder for the cyclic bending.

\subsection{Testing procedure}


The cyclic loading tests were performed using the prepared cantilever with a fully reversible strain, as outlined above. In the setup shown in figure 1 , the cantilever is actuated by a piezoelectric device (Physik Instrumente, Waldbronn, Germany), and a laser (wavelength of $635 \mathrm{~nm}$ ) is directed onto the vibrating cantilever. The reflected beam is detected by an area detector, which measures the reflected laser beam position and intensity. The cyclic displacement of the laser spot on the area detector caused by the vibrating cantilever is analyzed to control the maximum strain amplitude of the cantilever. The strain amplitude at the surface at the fixed end was determined to be $5.5 \times 10^{-3} \pm 2.8 \times 10^{-5}$ from finite element simulation (according to the thickness of the cantilever and to the measured maximum deflection of $10 \mathrm{~mm}( \pm 0.3 \mathrm{~mm}))$.

Figure 3(a) gives an according overview of the sample by applying UV LSM. The surface roughness (i.e. the height of hill-valley) of the cantilever was analyzed with UV LSM measurements. The surface morphology and the sub-surface microstructure were systematically investigated using FIB by serial cross-sectioning. Also, the average hardness of the undamaged and damaged regions was investigated by over 100 times nanoindentation using continuous stiffness measurement (CSM) mode (G200, Agilent, Santa Clara, CA, USA) with a Berkovich indenter tip.

Finally, cross-sectional TEM lamellae were prepared by FIB from the undamaged and damaged regions. The final ion milling was done by keeping the Ga ion beam as low as 10pA. In TEM, the high-resolution imaging mode was utilized to investigate the damaged (coarsened) microstructures using a Tecnai F20 TEM (FEI Co., Hillsboro, OR, USA).

\section{Results}

\subsection{Evolution of surface damage}

The fatigue induced damage distribution is illustrated by a UV LSM micrograph in figure $3(\mathrm{a})$, which shows the surface of the nt-Cu cantilever after $1.3 \times 10^{6}$ cycles. The damage front 
has reached a distance of $\sim 1.2 \mathrm{~mm}$ from the fixed end, which is equivalent to the strain amplitude $\left(\varepsilon_{\mathrm{a}}\right)$ of $\varepsilon_{\mathrm{a}}=4 \times 10^{-3}$ (see figure 2). For reference, the calculated maximum strain amplitude is $\varepsilon_{\mathrm{a}}=5.5 \times 10^{-3}$ at the fixed end of the cantilever.

From systematic SEM observations of the damage morphology, the damaged zones in the cantilever can be roughly classified into five regions as shown in figure 3(a). The surface strain amplitude $\left(\varepsilon_{\mathrm{a}, \text { surface }}\right)$ corresponding to each region was estimated using the simulation data from figure 2. Region with $\varepsilon_{\mathrm{a} \text {,surface }}<\sim 4 \times 10^{-3}$ represents the undamaged surface (see figure $3(\mathrm{~b})$ ), indicating that the critical $\varepsilon_{\mathrm{a} \text {,surface }}$ for the initiation of the surface damage may be $\varepsilon_{\mathrm{a} \text {,surface }}=\sim 4 \times 10^{-3}$ at the least for the given number of cycles. Region with $\sim 4 \times 10^{-3}<$ $\varepsilon_{\mathrm{a}, \text { surface }}<\sim 4.2 \times 10^{-3}$ shows a few damage sites that seemingly develop randomly. In region with $\sim 4.2 \times 10^{-3}<\varepsilon_{\mathrm{a} \text {,surface }}<\sim 4.4 \times 10^{-3}$, surface features were developed more homogeneously and small cracks were initiated (Fig. 3(c)). In region , with $\sim 4.4 \times 10^{-3}<\varepsilon_{\mathrm{a} \text {,surface }}<\sim 4.8 \times 10^{-3}$, propagation and/or coalescence of cracks is observed together with a homogeneously damaged surface. Region with $\sim 4.8 \times 10^{-3}<\varepsilon_{\mathrm{a} \text {,surface }}<5.5 \times 10^{-3}$ exhibited long cracks, i.e. extending over many grains (Fig. 3(d)), that led to the final failure of the cantilever, as marked by the black arrow in figure $3(\mathrm{~d})$.

Interestingly, figure 3(c) shows that the crack initiation is predominantly developed at an angle of $\sim \pm 45^{\circ}$ with respect to the loading direction. The long cracks, however, propagate almost perpendicular to the loading direction as shown in figure 3(d). The surface morphology of the damaged regions show randomly oriented features, which are different from previously reported slip bands with an angle of $45^{\circ}$ relative to the loading axis [27-29]. Figure 4(a) shows that these features are related to the "hill-valley" morphology. The damage surface profile is different from the "extrusion-intrusion" morphology that is typically observed in conventional metallic material due to fatigue [32]. For instance, fatigued polycrystalline $\mathrm{Cu}$ exhibits surface extrusions or intrusions, which are related to persistent slip bands [32]. In contrast, nt metals have been shown to have a different damage morphology 
with so-called "depression" areas [28-29]. The height of this morphology is defined as the altitude difference between an adjacent "hill" and "valley". An example of the cross-sectional measurement of the height by using UV LSM in region of figure 4(a) is shown in figure 4(b). The height of depressions, in the region $(\sim 70 \mathrm{~nm})$, is larger than that of the undamaged ( $40 \mathrm{~nm}$ ) region shown as in figure 3(a), which is comparable to the result obtained from tension-tension fatigue of $\mathrm{nt} \mathrm{Cu}$ [28-29]. In the damaged region, the depression height shows a clear linear relation with $\varepsilon_{\mathrm{a} \text {,surface }}$ (Fig. 4(a)). Interestingly, the crack propagation region ( in figure $4(\mathrm{a})$ ) indicates a saturation in depression height for $\varepsilon_{\mathrm{a} \text {,surface }}$ (from $\sim 180$ to $\sim 210 \mathrm{~nm}$ ).

\subsection{Subsurface microstructure change}

The microstructural changes are shown in the cross-sectional TEM micrographs taken from the undamaged and the crack initiation zones, region and , respectively, in figure 5. The TEM samples were taken from directly under the surface. Figure 5(a) shows the representative microstructure of an undamaged region with densely-stacked nanotwins with a mean thickness of $\sim 25 \mathrm{~nm}$. The twin stacks have long $\Sigma 3\left\{\begin{array}{lll}1 & 1 & 1\end{array}\right\}$ coherent twin boundaries (CTBs) normal to the growth direction. It is also seen that the $\mathrm{nt} \mathrm{Cu}$ has grown in columnar domains, which have a typical size of about $1 \mu \mathrm{m}$. These domains are bordered by $\Sigma 3\left\{\begin{array}{lll}1 & 1 & 2\end{array}\right\}$ incoherent twin boundaries (ITBs) [20-21]. In figure 5(a) the CTBs are marked with white dashed lines and ITBs are marked with red dashed lines. Fig. 5(b) shows the microstructure in the detwinned zone at $\varepsilon_{\mathrm{a} \text {,surface }}=\sim 4.4 \times 10^{-3}$ (region ). Clearly, coarsening of the $\mathrm{nt}$ microstructure, both in thickness and width, is observable. CTBs are still perpendicular to the growth direction. However, some of the CTB contain fairly large steps, which may be related to collective glide of Shockley partial dislocations on the CTBs and on the adjacent $\left\{\begin{array}{lll}1 & 1 & 1\end{array}\right\}$ glide planes of the ITBs, presumably leading to detwinning and coarsening by twin boundary migration [20-21]. 
Figure 6 shows representative SEM micrographs of the subsurface microstructure prepared by a serial cross-sectioning. Here, the transition from undamaged to fully damaged regions can be observed. It is evident that, with increasing $\varepsilon_{\mathrm{a} \text {,surface, }}$ the degree of detwinning increases as well. In the region between and , some twin boundaries and domains are no longer straight but the initial twin boundary orientation has survived. Underneath the localized depression zone in figure 6(b), detwinned structures (merged twin boundaries and domains) show a decrease in thickness from the surface to the interior of the sample. Crack initiation sites can be found under deeply depressed regions, see e.g. black arrows in figure 6(c). The crack is propagating perpendicular to the surface branching as it interacts with twin boundaries as shown in figures 6(d) and (e). With increasing penetration depth the cracks bend into a parallel direction relative to the twin boundaries and surface respectively.

The quantitative analyses of domain width and detwinned zone thickness (see figure $7(\mathrm{a}))$ in the main cantilever axis are plotted versus the $\varepsilon_{\mathrm{a}, \text { surface }}$ in figure $7(\mathrm{~b})$. The detwinning process leads to a noticeable increase in the detwinned zone thickness, from an originally $25 \mathrm{~nm}$ twin thickness to $\sim 2.5 \mu \mathrm{m}$. The domain width changed from originally $1 \mu \mathrm{m}$ to a length of $\sim 17 \mu \mathrm{m}$. Interestingly, the size of the coarsened domain area seems to be well matched with the depression zone size generated as a function of $\varepsilon_{\mathrm{a} \text {,surface }}$. For example, the domain width in region is consistent with the depression zone size of $\sim 10 \mu \mathrm{m}$.

Furthermore, even the surface damage free zone near the crack initiation region exhibits well-developed detwinned microstructures (see the figure 7(b)). Consistently, the twin density measured parallel to the direction of the detwinning zone thickness shows a clear decrease with $\varepsilon_{\mathrm{a}, \text { surface, }}$, where it changes from $\sim 40 / \mu \mathrm{m}$ to $\sim 2 / \mu \mathrm{m}$. Moreover, as presented in figure 8(a) and (b), the twin thickness (TT) and the domain width (DW) in the coarsened zone are functions of the distance from the surface, since the strain amplitudes decreases towards the interior of the beam. 


\subsection{Mechanical properties}

The hardness and elastic modulus distribution obtained from CSM nanoindentation measurement in the region from to $\left(\right.$ for $\sim 3.85 \times 10^{-3}<\varepsilon_{\mathrm{a} \text {,surface }}<\sim 4.1 \times 10^{-3}$ ) are presented in figure 9(a). The indentations were made with a maximum depth of $h_{\max }=\sim 700 \mathrm{~nm}$ and constant strain rate, $\mathrm{d} \varepsilon / \mathrm{d} t=h^{-1}(\mathrm{~d} h / \mathrm{d} t)=0.05 / \mathrm{s}$. No significant decrease in the elastic modulus is observed ( $\sim 125 \mathrm{GPa}$ in agreement with previous results [2]) although a clear change in the hardness value is measured. Additionally, there is a considerable gradient in hardness distribution in the damage initiation zone. The average hardness of region is lower (by $\sim 30$ \%) than that of region. The average hardness (estimated according to the Oliver-Pharr method [33-34]) of region and is about 3.11 and $2.13 \mathrm{GPa}$, respectively, in agreement with previous results for nt [25] and ultra-fine grained metals [5]. This hardness difference coincides with areas where the detwinning induced microstructural changes. The tendency for softening with increasing twin thickness is in good agreement with grain size dominated strengthening in the conventional polycrystalline counterpart [3-4]. The representative images in figures 9(b) and (c) show the microstructure in the indentation zone. The cross-sectional SEM micrograph of region shows severe deformation mainly in the coarsened structure near the indentation. In comparison indentation in region leads to less coarsening and only some CTB structures are detwinned, especially parallel to the CTBs.

\section{Discussion}

\section{Evolution of surface damage}

It is well known in fatigue studies that cracks are initiated at the surface of the specimen. Thus, the surface quality has a major influence on the fatigue behavior and lifetime. For our samples, the initial surface is fairly smooth with a roughness of less than $50 \mathrm{~nm}$ (see Fig. 4a). Therefore, it is not surprising that no immediate crack formation starting from the 
surface was observed. In contrast, the surface morphology evolved during cyclic loading, and after reaching a certain roughness cracks formed. The surface damage evolution can be observed clearly by the present experiments and is consistent with the literature as cyclic loading induces surface depressions in $\mathrm{nt} \mathrm{Cu}$. Pan et al. argue that the depression areas represent softer regions which then undergo larger strain excursions than the surrounding material [27]. Other authors reported that a set of depressions is predominantly initiated at a domain (or columnar grain) [24,27-28]. Such domains can be discerned in a cross section as columnar stacks of twins (Fig. 5(a) and [27]) and have a typical diameter of about $1 \mu \mathrm{m}$. In this study, the depressions were observed to be related to the applied strain amplitude at the surface, $\varepsilon_{\mathrm{a} \text {,surface }}$ as the depressed regions become wider and deeper with increasing amplitude (see regions and of figure 4(a)). In the severely damaged region ( of figure 4(a)), many "hills" and "valleys" are distributed homogeneously covering the entire surface. This surface morphology indicates already that the damage propagation is not limited to a specific domain, in the original microstructure. However, as discussed below, the domains grow substantially during cyclic loading. .

\section{Qualitative observations of subsurface microstructural changes}

In conventional metals, detwinning can occur via the elimination of growth twins [2022]. In the $\mathrm{nt} \mathrm{Cu}$, the twin density is much higher and detwinning occurs in a systematic manner depending on the applied cyclic strain. It can be argued that the detwinning under these conditions might be the result of partial dislocation migration along the CTBs and ITBs [20-22]. If the migration of partial dislocation is related to the accumulated plastic strain during cycling, it could be expected that an increase in TT and DW with increasing strain amplitude and cycle number could be observed. Indeed this has been observed, as the resulting twin thickness is the largest at the surface where the strain amplitude reaches maximum (see figure $8(\mathrm{a})$ ). For example, after $N=1.3 \times 10^{6}$ cycles, the TT at the surface 
increases up to $\sim 120 \mathrm{~nm}$ and $\sim 920 \mathrm{~nm}$ for strain amplitudes of $\varepsilon_{\mathrm{a} \text {,surface }} \sim 3.85$ and $5.5 \times 10^{-3}$, respectively. This increase of the size by more than an order of magnitude is clearly larger than that observed after a tension-tension $\left(\mathrm{R}_{\varepsilon}=\sim 0\right.$ or 0.1$)$ fatigue test, where the average thickness, 40-55 nm, was merely two times that of the original size [28]. Shute et al. used a rather large specimen and eventually did not find the site with the largest twins. It can be speculated that during a tension-tension experiment, a fatal failure might occur when one site develops large enough twins that allows a crack to develop. However, it is challenging to locate the relevant spot after the experiment. In our studies, the cyclic loading is applied in a strain controlled fashion preventing local failure. Therefore, the detwinning process can be studied systematically, and the large gradients in strain amplitude allow us to correlate damage and microstructural evolution to strain amplitude and number of cycles.

Furthermore, the DW at the surface increased to $\sim 17 \mu \mathrm{m}$ at a strain amplitude $\varepsilon_{\mathrm{a} \text {,surface }}$ of $\sim 5.5 \times 10^{-3}$ from the original domain width of $\sim 1 \mu \mathrm{m}$, as shown in figure $8(\mathrm{~b})$. In contrast, no evidence for coarsening of the DW in tension-tension fatigue has been found [13,27-29]. There are two possible explanations to explain the difference. First, more potential sites for partial dislocation migrations are activated under the tension-compression strain ratio of $\mathrm{R}_{\varepsilon}=$ -1 in this study [35]. Second, the expected back and forth slip of partial dislocations under $\mathrm{R}_{\varepsilon}$ $=-1$ leads to a more pronounced detwinning behavior, i.e. increase in TT, than under $\mathrm{R}_{\varepsilon} \sim 0$ or 0.1 conditions [35]. Both mechanisms may favor the subsequent DW coarsening if the motion of the ITBs between domains is hindered by the CTBs between the nanotwins.

\section{Quantitative analysis of the microstructural changes}

Under cyclic bending conditions, as cantilever strain is related to the bending moment, it is possible to determine strain at each position along the beam by the curvature relation [36], $\varepsilon=\mathrm{y} / \mathrm{R}$. Here, $\varepsilon$ is the bending strain, $\mathrm{y}$ is a distance from neutral axis to the detwinning boundary of the detwinned structure, and $\mathrm{R}$ is the curvature of cantilever at the fixed end. 
During cyclic bending, the smallest radius relates to the strain amplitude, $\varepsilon_{\mathrm{a}}$, which is constant as long as the resonance frequency does not change. $\varepsilon_{\mathrm{a}}$ varies as a function of $\mathrm{y}$, thus TT and DW in the detwinned zone do not vary only along the length of the beam but also into the depth of the materials (see figure 8(a) and (b)).

From Fig. 7, it is obvious that the damage initiation is observed at a strain amplitude of $\sim 4 \times 10^{-3}$ while cracks are only observed for amplitudes larger than $\sim 4.4 \times 10^{-3}$. Therefore, detailed studies of the microstructure and damage morphology were carried out in this strain amplitude regime. Changes in TT and DW are measured and the normalized size with respect to original size of TT and DW as a function of $\varepsilon_{\mathrm{a}}$ for $1.3 \times 10^{6}$ cycles are summarized in figure 10. Interestingly, both TT and DW in the uncracked region appear to be linearly dependent of $\varepsilon_{\mathrm{a}}$. This implies that $\varepsilon_{\mathrm{a}}$ is governing the amount of detwinning, which is linearly proportional to the migration of detwinning dislocation as described in Ref. 37. The solid lines for the changes in TT and DW are fitted for the uncracked regime (open symbols). The critical strain amplitudes, $\varepsilon_{\mathrm{a}} \sim 3 \times 10^{-3}$ and $\varepsilon_{\mathrm{a}} \sim 3.7 \times 10^{-3}$, are found, respectively, for the initiation of detwinning and domain growth at the intersection of these lines with the solid line in the figure representing normalized values of 1. For the fit, data points (full symbols) obtained for the crack propagation region (i.e., $\varepsilon_{\mathrm{a} \text {,suface }}>\sim 4.4 \times 10^{-3}$ ) were not included. However, it seems that the twins still coarsen with increasing strain amplitude while DW saturates. This may be related to the fact that less plastic energy is dissipated when cracks are formed and propagated.

As shown in figure 10 , the dimensions of TT and DW vary as a function of $\varepsilon_{\mathrm{a}}$, and accordingly the detwinning behavior of $\mathrm{nt} \mathrm{Cu}$ can be divided into four distinct regimes. For the given number of cycles of $1.3 \times 10^{6}$, a $\varepsilon_{\mathrm{a}}$ of $\sim 3 \times 10^{-3}$ is necessary for the initiation of detwinning, this marks the end of regime -a, of the "undamaged region". This regime is characterized by neither depressions nor changes to the TT or DW. It can be expected that regime $-b$ is characterized by twin thickening, by removing CTBs presumably by motion of 
partial dislocations. At $\varepsilon_{\mathrm{a}}$ of $\sim 3.7 \times 10^{-3}$, the regime - shows coarsening of both twins as well as domains, the latter being related to the motion of ITBs. Here, prevailing localized depression morphologies are observed. Finally, the regime - is defined as the crack propagation regime. The estimated $\varepsilon_{\mathrm{a}}$ of $\sim 4.4 \times 10^{-3}$ represents the initiation of "cracking" with fully developed depressions. While these different stages of fatigue damage were observed at the same cycle number with different strain amplitudes, it is reasonable to assume that the stages - represent the typical sequence of fatigue mechanisms in $\mathrm{nt} \mathrm{Cu}$ as a function of cycle number at a given strain amplitude.

\section{Crack propagation}

As described above, regime - is characterized by cracking under the deeply depressed zones. In the present study, a significant transition from transgranular crack propagation under mode I (perpendicular to the surface and the loading direction) to intergranular crack propagation in a mode II orientation can be observed; the crack branches at twin boundaries and therefore seems to behave differently compared to conventional fcc metals in which fatigue cracks typically do not branch [32]. The same is true for nt $\mathrm{Cu}$ fractured under static tensile loading [28]. To further understand the behavior, it is necessary to establish a criterion that differentiates transgranular from intergranular crack propagation. A possible explanation for the change in crack propagation mode could be the existence of the twin thickness gradient between the detwinned material close to and away from the surface. Previous studies showed that TBs could serve as crack path due to the elastic anisotropy of TBs, i.e. elastic incompatibility of TBs [38-39]. Thus the transition of cracking to mode II indicates that TBs might serve as a preferential site for the crack propagation [27-29]. Overall, a comparison between the fatigue crack propagation in nt and nc metals shows that crack propagation in nc metals along grain boundaries seems to be fairly easy and is accompanied 
by grain coarsening in front of the crack tip $[16,18]$. In contrast, nanotwins seem to be more stable as the crack does not propagate perpendicular to the CTB but is deflected. Also, near the crack tip no additional coarsening of the microstructure is observed.

\section{Conclusions}

We have presented a methodology for fatigue testing based on a vibrating beam setup, which allows for the investigation of the damage evolution in thin foil samples. A detailed study of microstructural changes and damage evolution for nanotwinned $\mathrm{Cu}$ has been carried out. High-purity $\mathrm{Cu}$ samples containing parallel columns of highly aligned nanotwins have been subjected to tension-compression cyclic loading. The systematic investigation of changes of the surface/subsurface microstructure has provided new insights into the influence of the applied strain on the detwinning evolution. One of the most significant results of the study is that the nanotwinned microstructure was found to coarsen with respect to both twin thickness as well as domain size as a function of strain amplitude. Surface damage in the form of depressions occurred as a result of the detwinning. Lateral size and height of the depressions increase with increasing strain amplitude and are well correlated with coarsening of twin domains. Eventually, the detwinned material mediates cracking in the severely depressed zone, which naturally occurs more frequently as the strain amplitude increases. Due to the orientation of the nanotwins with respect to the loading direction, fatigue cracks tend to branch and change in their modality.

\section{Acknowledgements}

BGY is grateful to the Alexander von Humboldt Stiftung for a postdoctoral Fellowship. XZ and YL acknowledges financial support by DoE-OBES under grant no. DE-SC0010482 for studies on mechanical behavior of nanotwinned metals. CE acknowledges support from DFG EB 362/3-1, SFB499 N01 and Fraunhofer Attract. 


\section{References}

1. Christian JW, Mahajan S. Prog Mater Sci 1995;39:1.

2. Weertman JR, Farkas D, Hemker K, Kung H, Mayo M, Mitra R, Van Swygenhoven H. MRS Bull 1999;24:44.

3. Kumar KS, Van Swygenhoven H, Suresh S. Acta Mater 2003;51:5743.

4. Meyers MA, Mishra A, Benson DJ. Prog Mater Sci 2006;51:427.5. Dao M, Lu L, Asaro RJ, De Hosson JTM, Ma E. Acta Mater 2007;55:4041.

6. Gu P, Dao M, Suresh S. Acta Mater 2014;67:409.

7. Zhang X, Misra A, Wang H, Nastasi M, Embury JD, Mitchell TE, Hoagland RG, Hirth JP. Appl Phys Lett 2004;84:1096.

8. Zhang X, Misra A, Wang H, Lima AL, Hundley MF, Hoagland RG. J Appl Phys 2005;97:094302.

9. Lu L, Shen Y, Chen X, Qian L, Lu K. Science 2004;304:422.

10. Lu L, Chen X, Huang X, Lu K. Science 2009;323:607.

11. Zhang X, Wang H, Chen XH, Lu L, Lu K, Hoagland RG, Misra A. Appl Phys Lett 2006;88:173116.

12. Zhu YT, Liao XZ, Wu XL. Prog Mater Sci 2012;57:1.

13. Zhang X, Misra A. Scripta Mater 2012;66:860.

14. Beyerline IJ, Zhang X, Misra A. Ann Rev Mater Res 2014;44:15.1

15. Balluffi RW, Cahn JW. Acta Metall 1981;29:493.

16. Legros M, Gianola DS, Hemker KJ. Acta Mater 2008;56:3380.

17. Lohmiller J, Baumbusch R, Kraft O, Gruber PA. Phy Rev Lett 2013;110:066101.

18. Padilla HA, Boyce BL. Exp Mech 2010;50:5.

19. Jin ZH, Gumbsch P, Albe K, Ma E, Lu K, Gleiter H, Hahn H. Acta Mater 2008;56:1126.

20. Li N, Wang J, Misra A, Zhang X, Huang JY, Hirth JP. Acta Mater 2011;59:5989.

21. Li N, Wang J, Huang JY, Misra A, Zhang X. Scripta Mater 2011;64:149.

22. Wang J, Li N, Anderoglu O, Zhang X, Misra A, Huang JY, Hirth JP. Acta Mater 2010;58:2262.

23. Lu N, Du K, Lu L, Ye Q. J Appl Phys 2014;115:024310. 
24. Li X, Wei Y, Lu L, Lu K, Gao H. Nature Mater 2010;464:877.

25. Bezares J, Jiao S, Liu Y, Bufford D, Lu L, Zhang X, Kulkarni, Asaro J. Acta Mater $12 ; 60: 4623$.

26. Wang YM, Sansoz F, LaGrange T, Ott RT, Marian J, Barbee Jr TW, Hamza AV. Nature Mater 2013;12:697.

27. Pan QS, Lu QH, Lu L. Acta Mater 2013;61:1383.

28. Shute CJ, Myers BD, Xie S, Li SY, Barbee Jr TW, Hodge AM, et al. Acta Mater 2011;59:4569.

29. Hodge AM, Furnish TA, Shute CJ, Liao Y, Huang X, Hong CS, Zhu YT, Barbee Jr. TW, Weertman JR. Scripta Mater 2012;66:872.

30. Burger S, Eberl C, Siegel A, Ludwig A, Kraft O. Sci Tech Adv Mater 2011;12:1.

31. Wang YC, Hoechbauer T, Swadener JG, Misra A, Hoagland RG, Nastasi M. Exp Mech 2006;46:503.

32. Zhang GP, Volkert CA, Schwaiger R, Wellner P, Arzt E, Kraft O. Acta Mater 2006;54:3127

33. Oliver WC, Pharr GM. J Mater Res 1992;7:1564.

34. Oliver WC, Pharr GM. J Mater Res 1004;19:3.28. Yu Q, Zhang J, Jiang Y, Li Q. Inter J Fat 2012;44:225.

35. Yu Q, Zhang J, Jiang Y, Li Q. Inter J Fat 2012;44:225.

36. Dieter GE. Mechanical Metallurgy. UK: McGraw-Hill; 1988.

37. Neumann P, Tonnessen A. In: Kettunen PO, Lepisto TK, Lehtonen ME, editors. Strength of metals and alloys. Oxford: Pergamon; 1988.

38. Heinz A, Neumann P. Acta Metall Mater 1990;38:1933.

39. Hutchinson JW. Suo Z. Adv Appl Mech 1992;29:63.

\section{List of Figures}


Figure 1. Schematic of cyclic bending test setups: Displacement amplitude acquisition by the area detector, where the displacement change obtained from the deflection of the cantilever is monitored by measuring the reflected laser spot on the area detector. Resonance frequency determination using the data of inset image, where the peak excitation frequency of the cantilever was selected.

Figure 2. The strain amplitude $\varepsilon_{\mathrm{a}}$ (under the maximum deflection of the cantilever versus the surface position along the cantilever) of the vibrating cantilever decreases along the cantilever with a maximum at the fixed end and almost zero strain amplitude at free end. Strain calculation conducted by FE simulation on a linear elastic copper cantilever $(1=8 \mathrm{~mm}, \mathrm{w}=$ $800 \mu \mathrm{m}, \mathrm{t}=\sim 20 \mu \mathrm{m})$ excited at its resonance frequency. The spheres at the free end represent the small mass attached to the cantilever during cyclic testing and the color code represents the calculated displacement at maximum deflection.

Figure 3. Representative micrographs for the surface damage of (a) fractured cantilever obtained from UV LSM measurement after cyclic bending for $\sim 1.3 \times 10^{6}$ cycles: (b) Undamaged, (c) onset of damage (crack initiation), and (d) severely damaged (crack propagation) regions obtained from SEM measurement. Especially, - were given in cantilever after fracture to define microstructure development from undamaged to fully damaged states according to change in the damage morphology.

Figure 4. (a) Fatigue induced damage forms as depression or hill-valley height variation as a function of $\varepsilon_{\mathrm{a} \text {,surface. }}$ Representative SEM micrographs in (a) illustrate the damage morphology at the surface. Error bars in (a) show the standard deviation for the individual height of $\sim 20$ hill-valleys. (b) Example of the measurement of depression or hill-valley height at the dashed arrow zone in of (a) with perpendicular dashed lines to $\mathrm{x}$-axis. ' $\mathrm{H}$ ' and ' $\mathrm{V}$ ' mean 'hill' and 'valley', respectively.

Figure 5. Representative TEM images of (a) the nanotwinned structure with $\sim 25 \mathrm{~nm}$ twin spacing in the undamaged region $\left(@ \varepsilon_{\mathrm{a}} \sim 3.3 \times 10^{-3}\right)$ and $(\mathrm{b})$ the representative detwinned microstructure in the damaged region $\left(@ \varepsilon_{\mathrm{a}} \sim 4.4 \times 10^{-3}\right)$. Evidence of crack initiation is prominent at microstructural steps (shown as white arrows) near the deep depressions. The domain width in (a) and the detwinning zone thickness in (b), respectively are highlighted.

Figure 6. The representative subsurface morphologies obtained from serial cross sectioning with increasing $\varepsilon_{\mathrm{a} \text {,surface: }}$ (a) undamaged zone in , (b) localized depression zone in , (c) crack initiation shown by black arrows in , (d) a representative crack propagation under the severely damaged zone in - (e) a magnified subset of (d) showing the crack branching and propagation parallel to CTBs.

Figure 7. (a) Representative subsurface morphology of the detwinned microstructure in showing detwinned zone thickness, detwinned twin thickness, and domain width. (b) Detwinned zone thickness and domain width as a function of $\varepsilon_{\mathrm{a} \text {,surface }}$ including damage initiation@ $\varepsilon_{\mathrm{a}, \text { surface }}=\sim 4 \times 10^{-3}$ and crack initiation @ $\varepsilon_{\mathrm{a} \text {,surface }}=\sim 4.4 \times 10^{-3}$ highlighted by dashed lines, respectively.

Figure 8. Change in the representative subsurface damage morphologies in the detwinned region: (a) twin thickness and (b) domain width as functions of $\varepsilon_{\mathrm{a}, \text { surface }}$ and distance from surface. 
Figure 9. Nanoindentation results from regions and : (a) Hardness and elastic modulus distribution. Magnified microstructural SEM images in the indentation zone of (b) the damaged region in and (c) the undamaged region in .

Figure 10. Summary of change in microstructure of detwinning region with $\varepsilon_{\mathrm{a}}$. Normalized twin thickness and domain width by their original size as a function of $\varepsilon_{\mathrm{a}}$ are shown. Experimental data are summarized with symbols: in damaged or undamaged zones (open)) and in crack propagation zone (closed). The detwinning is divided into four regimes described in the text. Regime -a represents the undamaged area, regime - $b$ indicates the initiation of twin thickness coarsening, regime - shows the initiation of domain width coarsening, and regime - with crack propagation. 

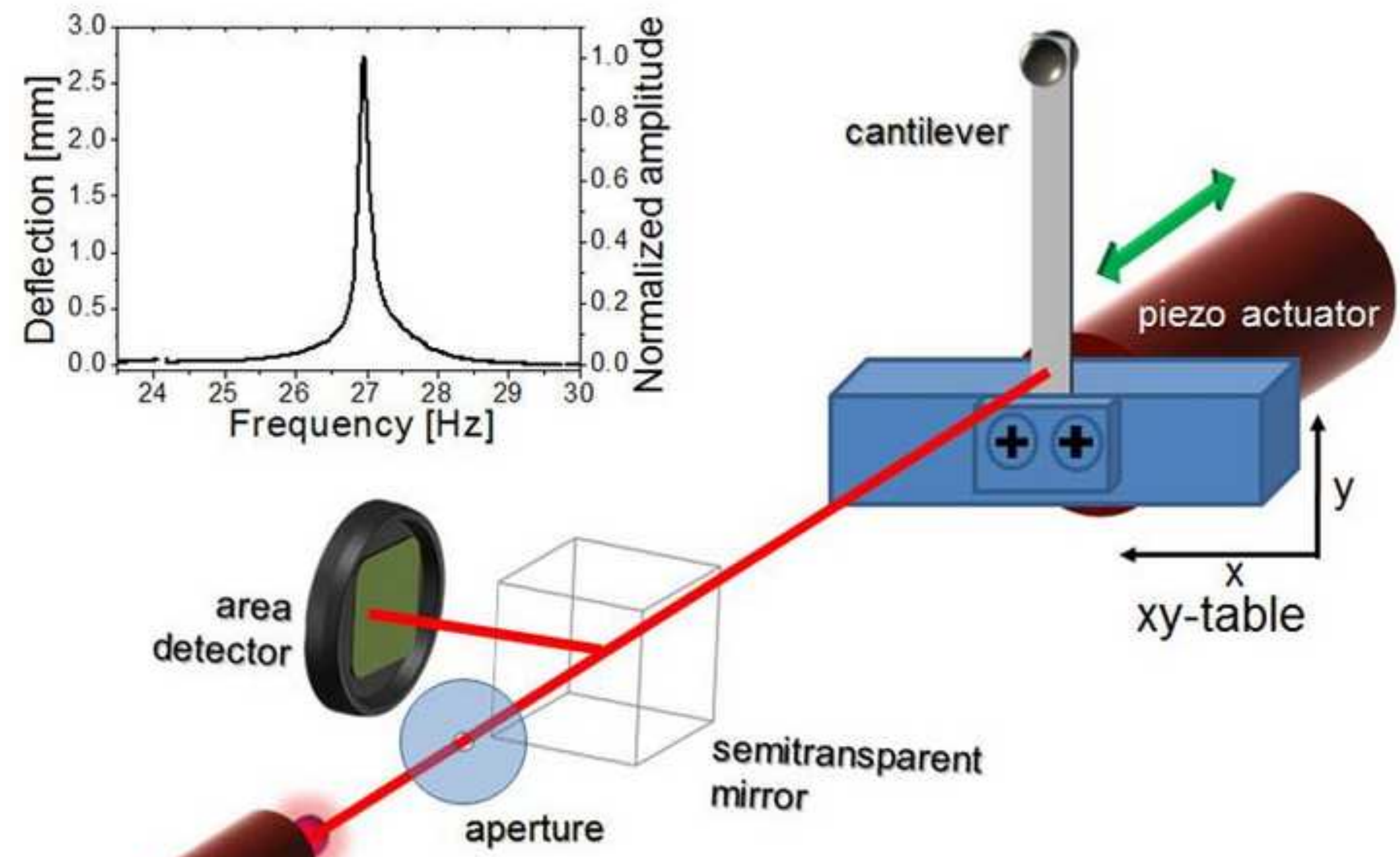

\section{laser}

\section{aperture}

semitransparent

detector

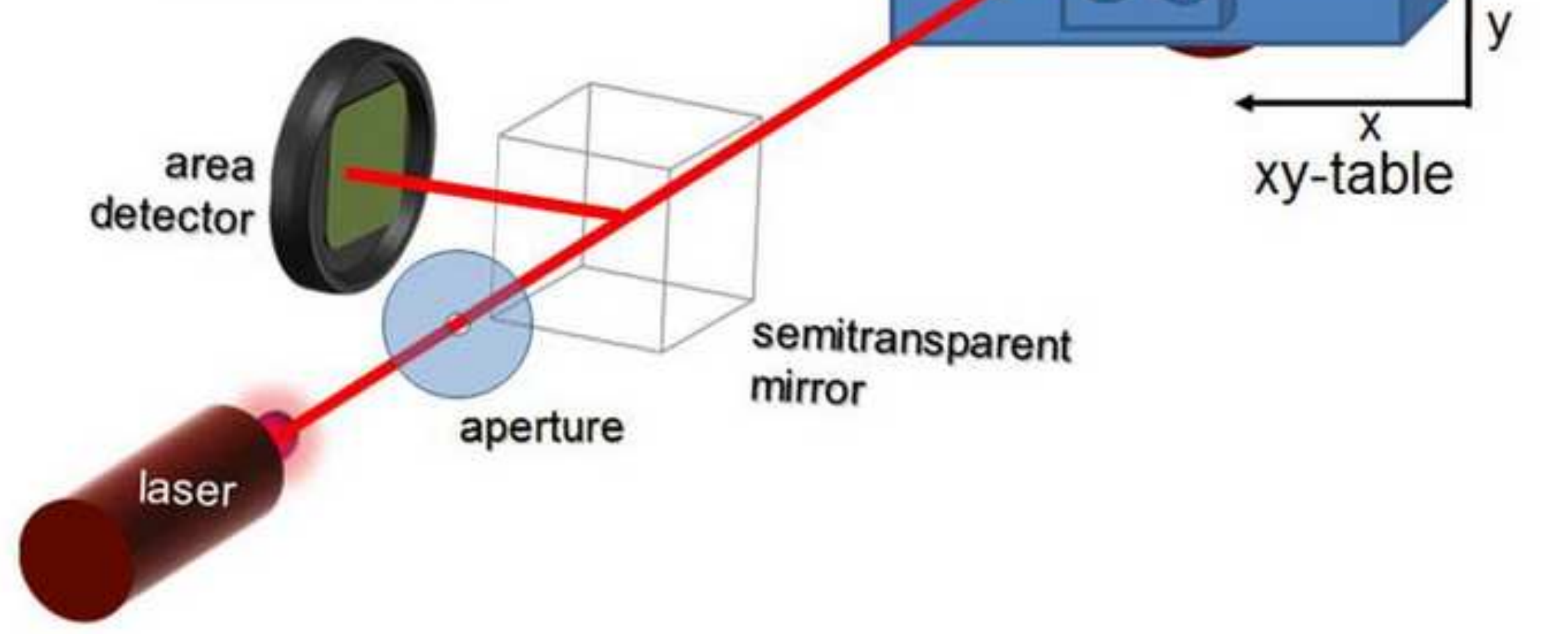




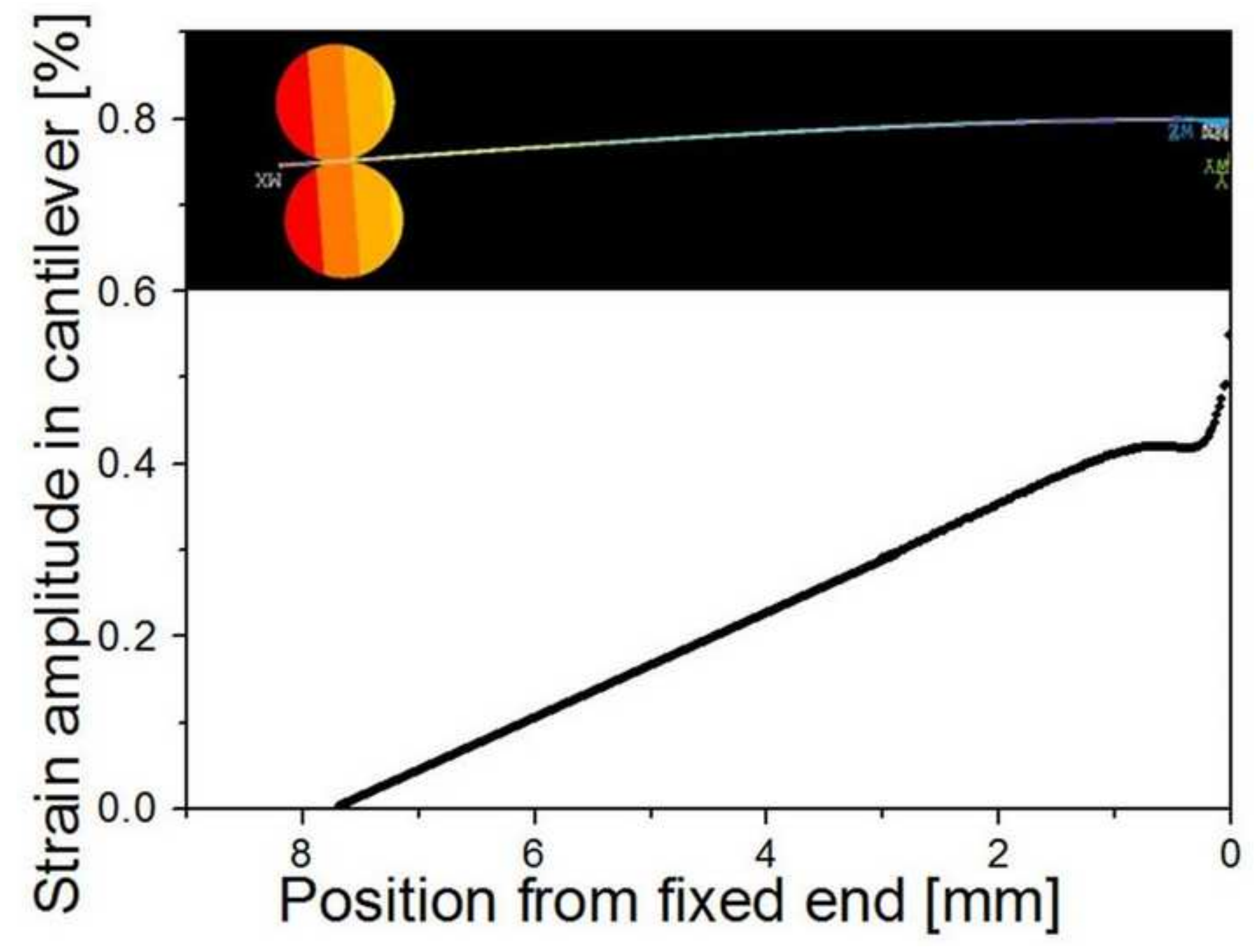

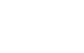


(a)

k. free end

(b)

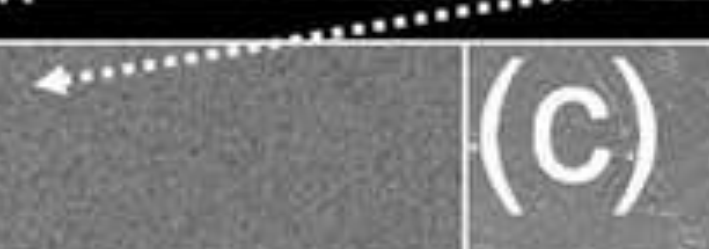

crack initiation

$\Rightarrow$

s.

(1)-(2) (3) (4) (5)
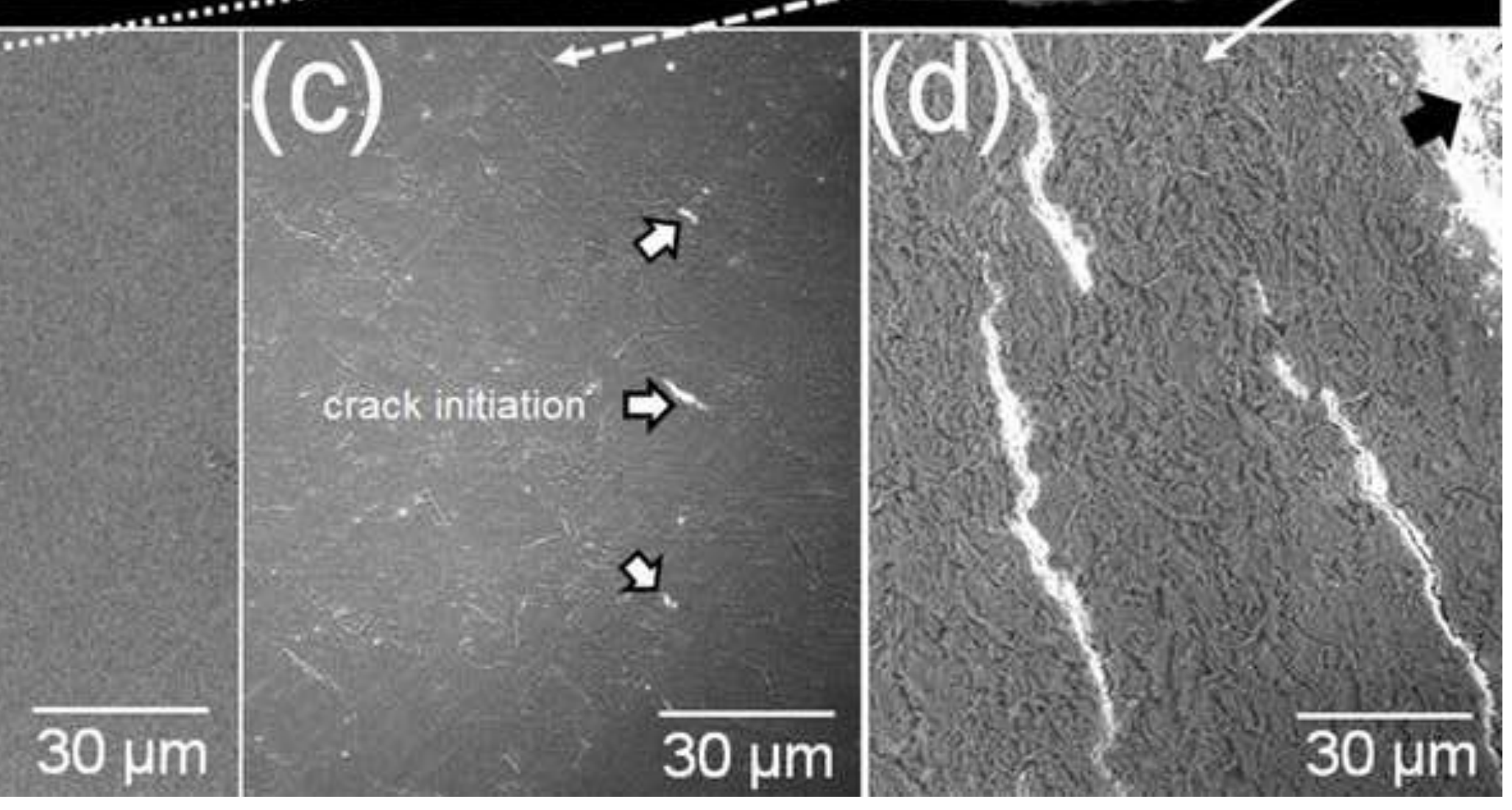

य

.

$\overline{30 \mu m}$

$30 \mu \mathrm{m}$ 


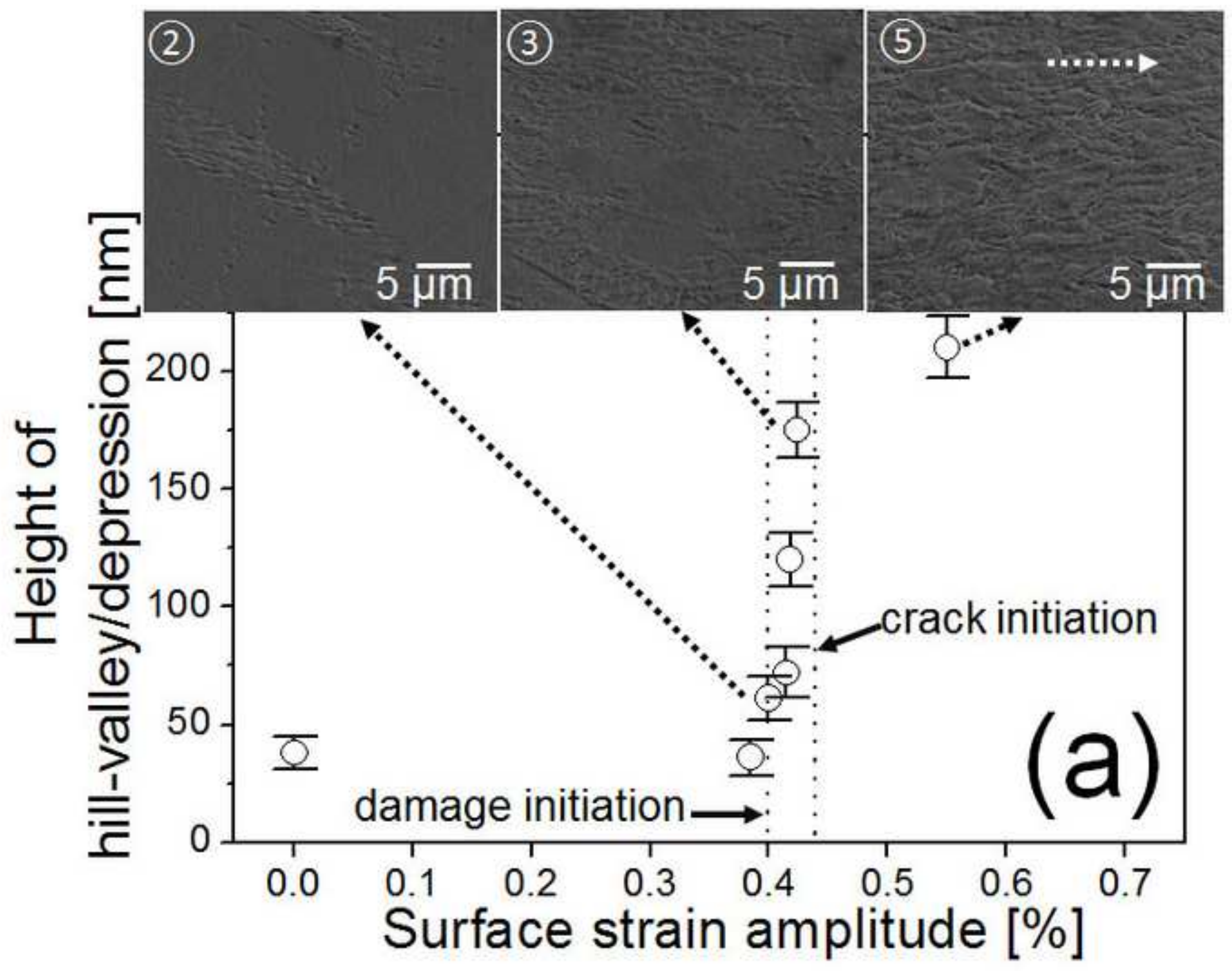




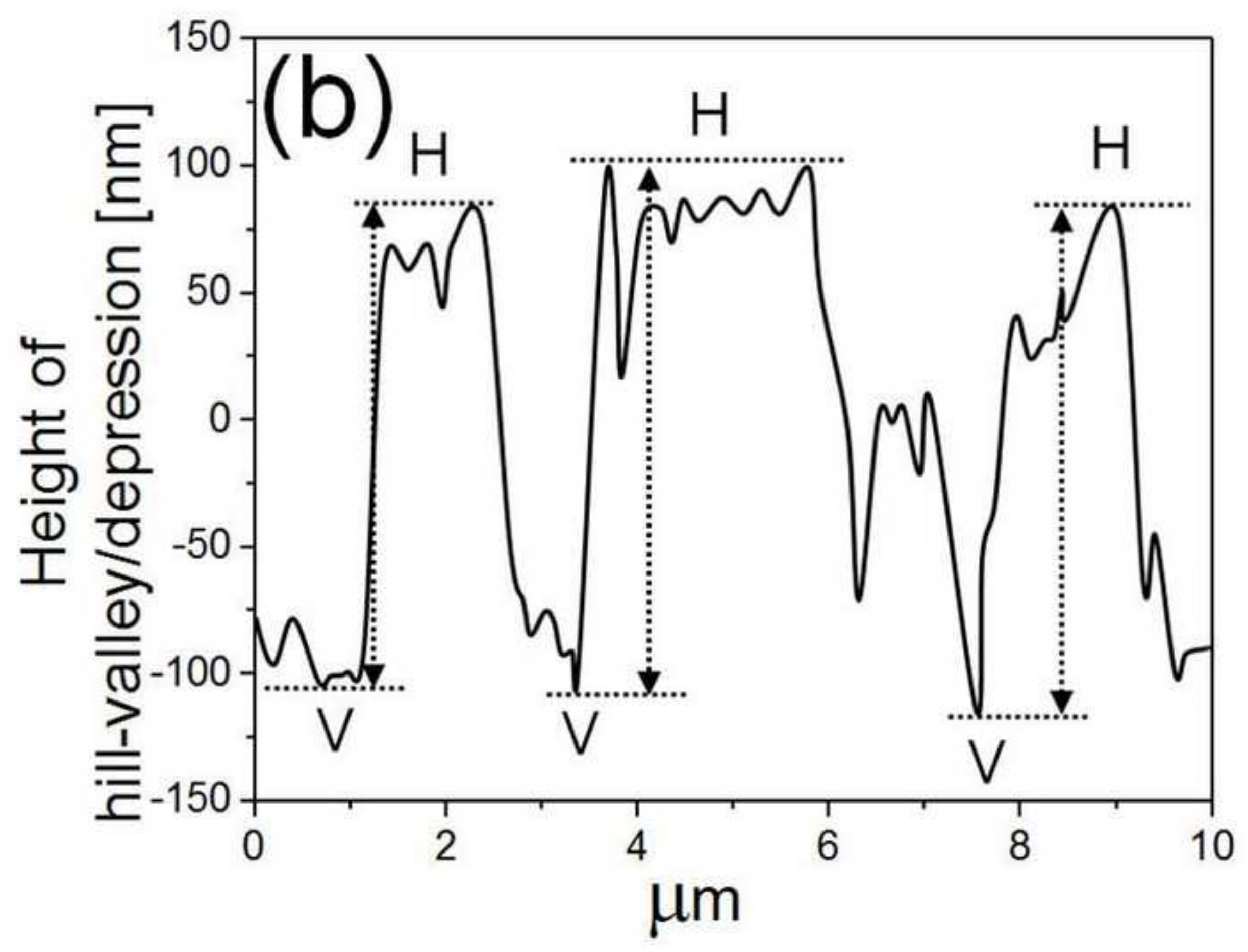




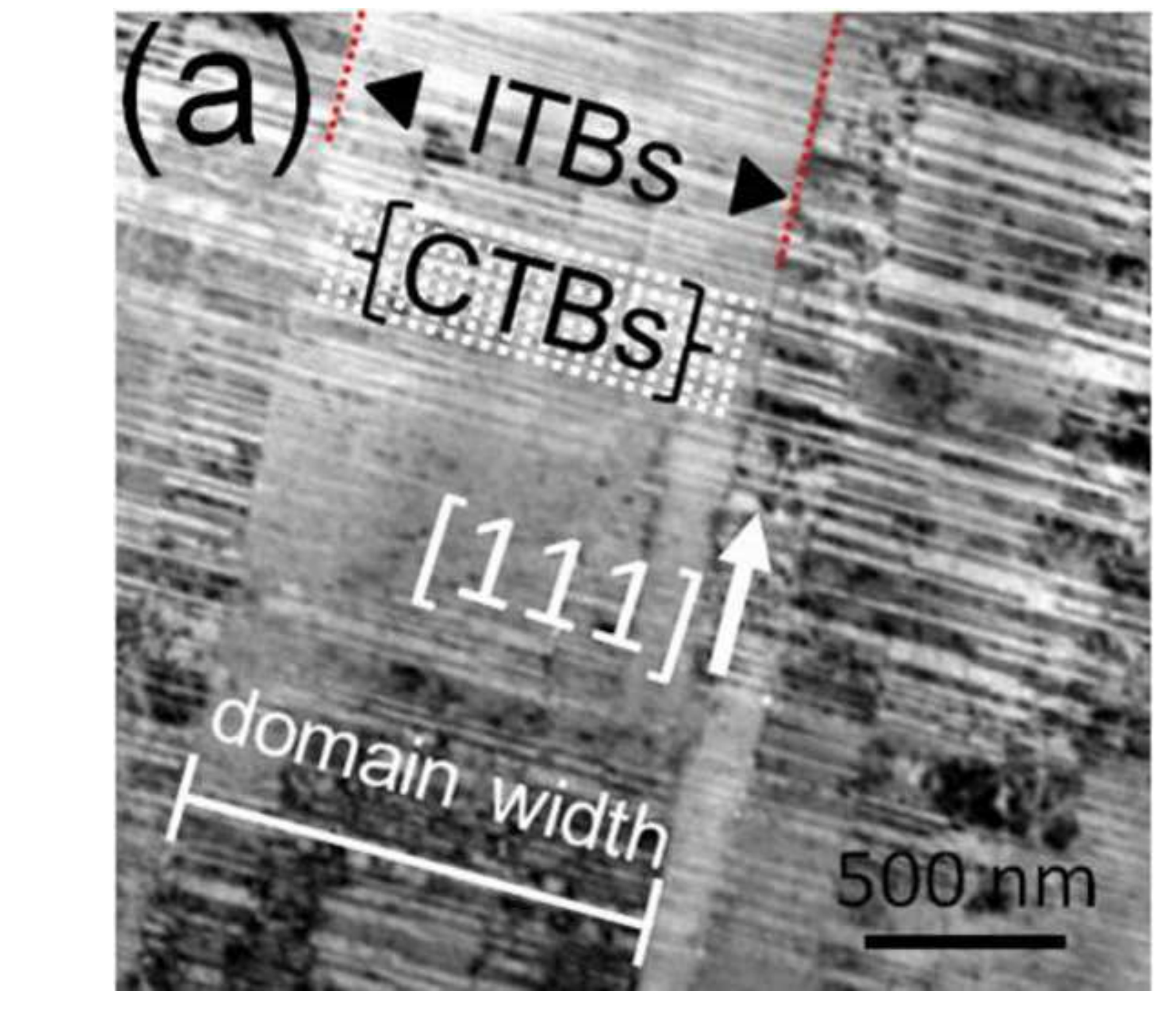

\section{.}
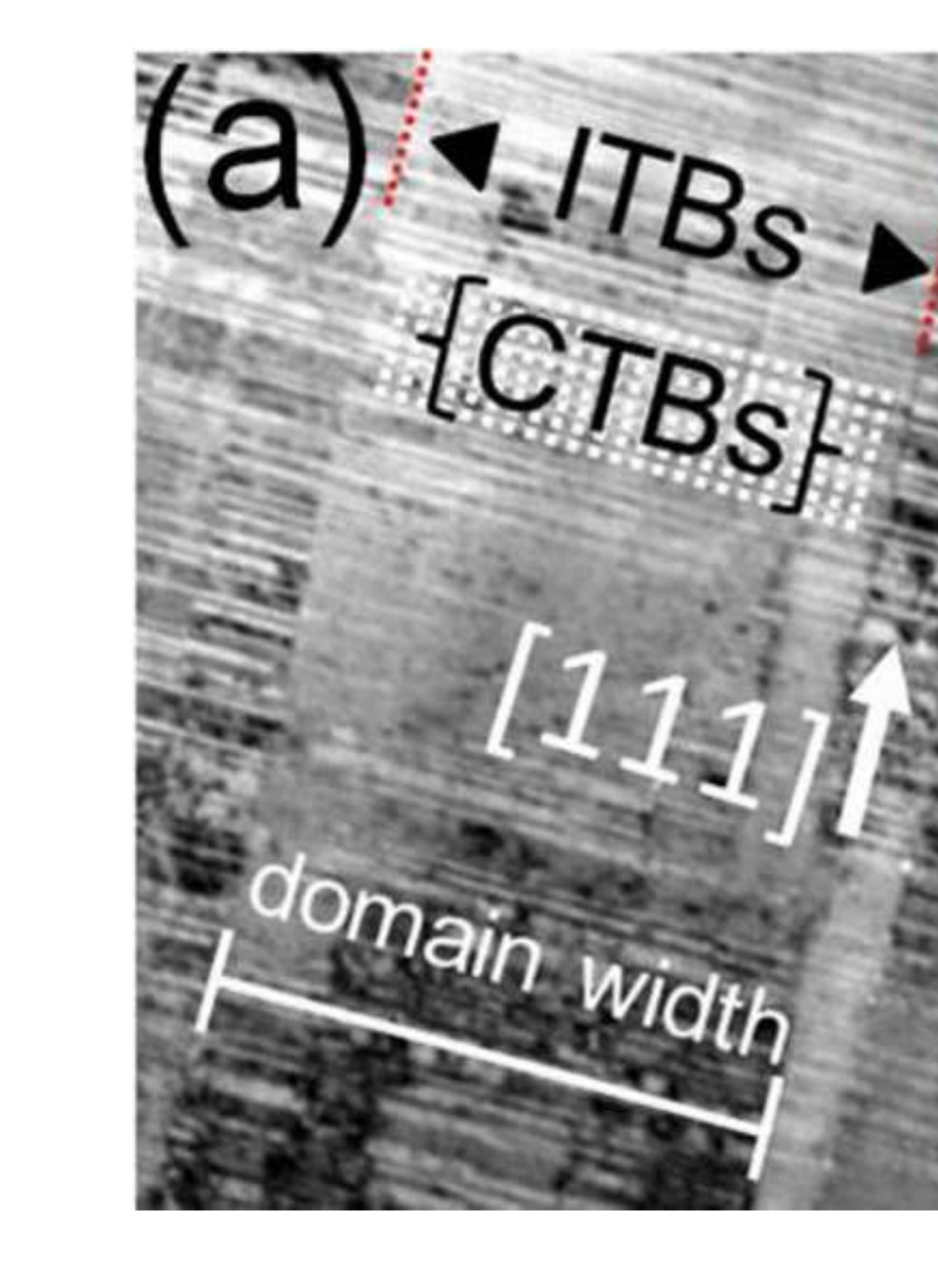

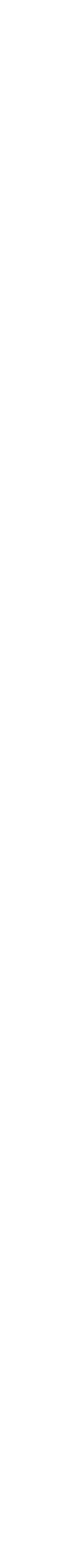

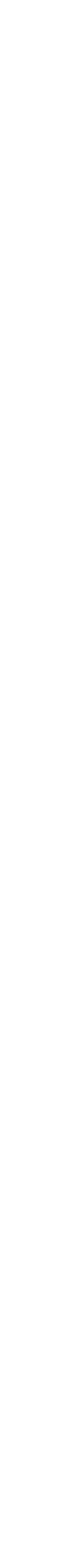

$$
\text { 촌 }
$$

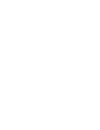




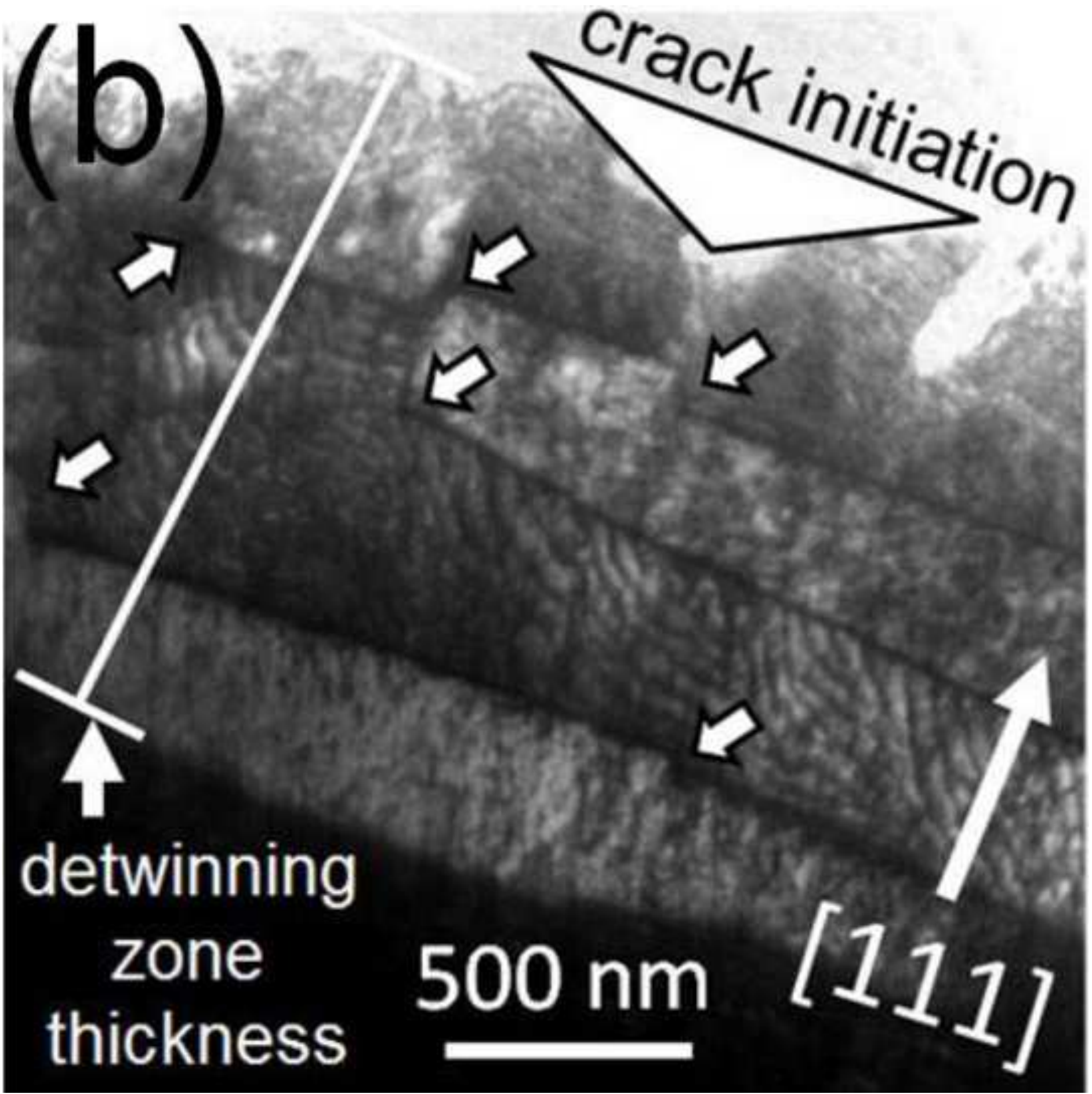




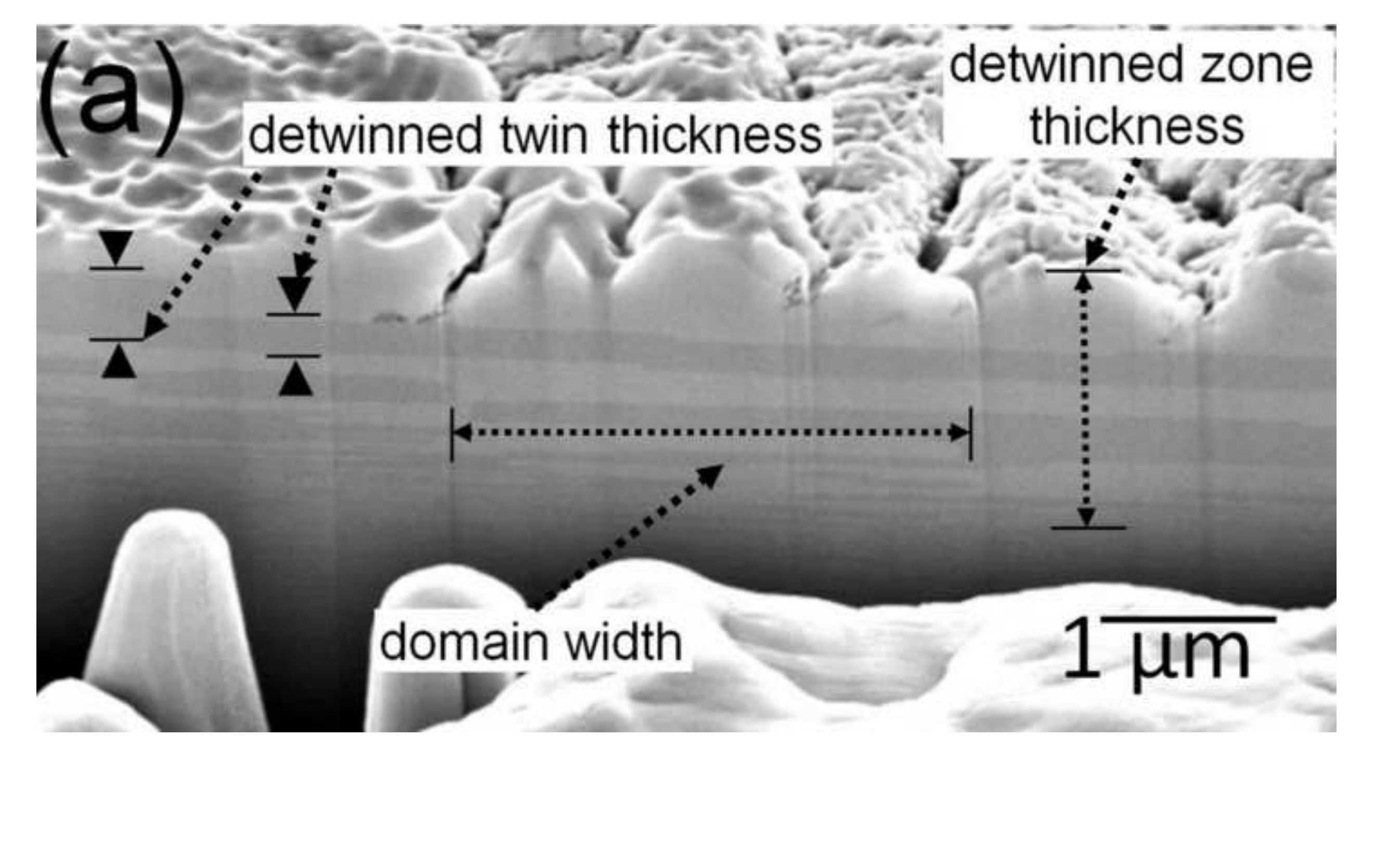
detwinned twin thickness thickness
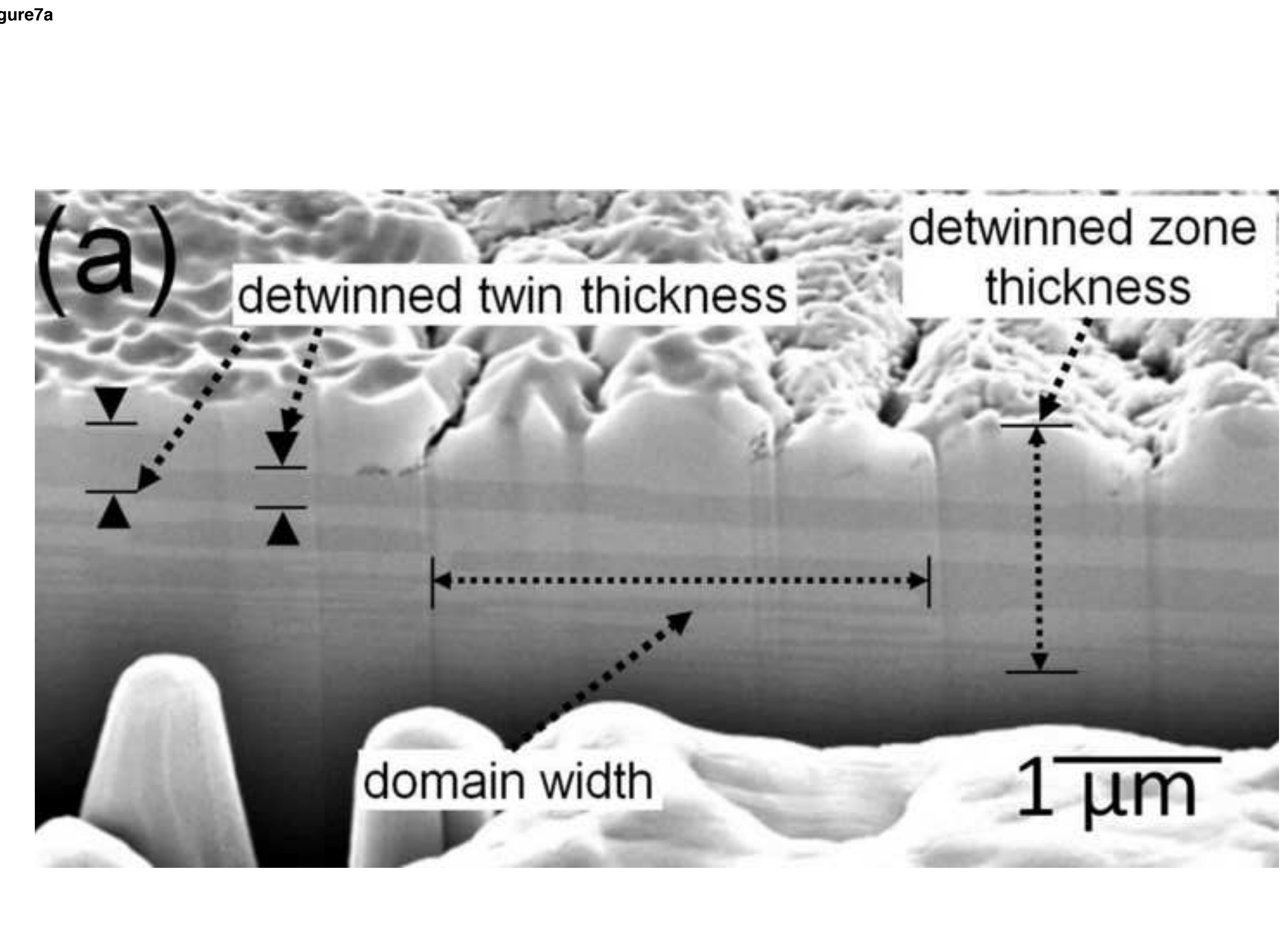

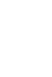
.

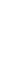

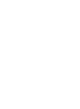

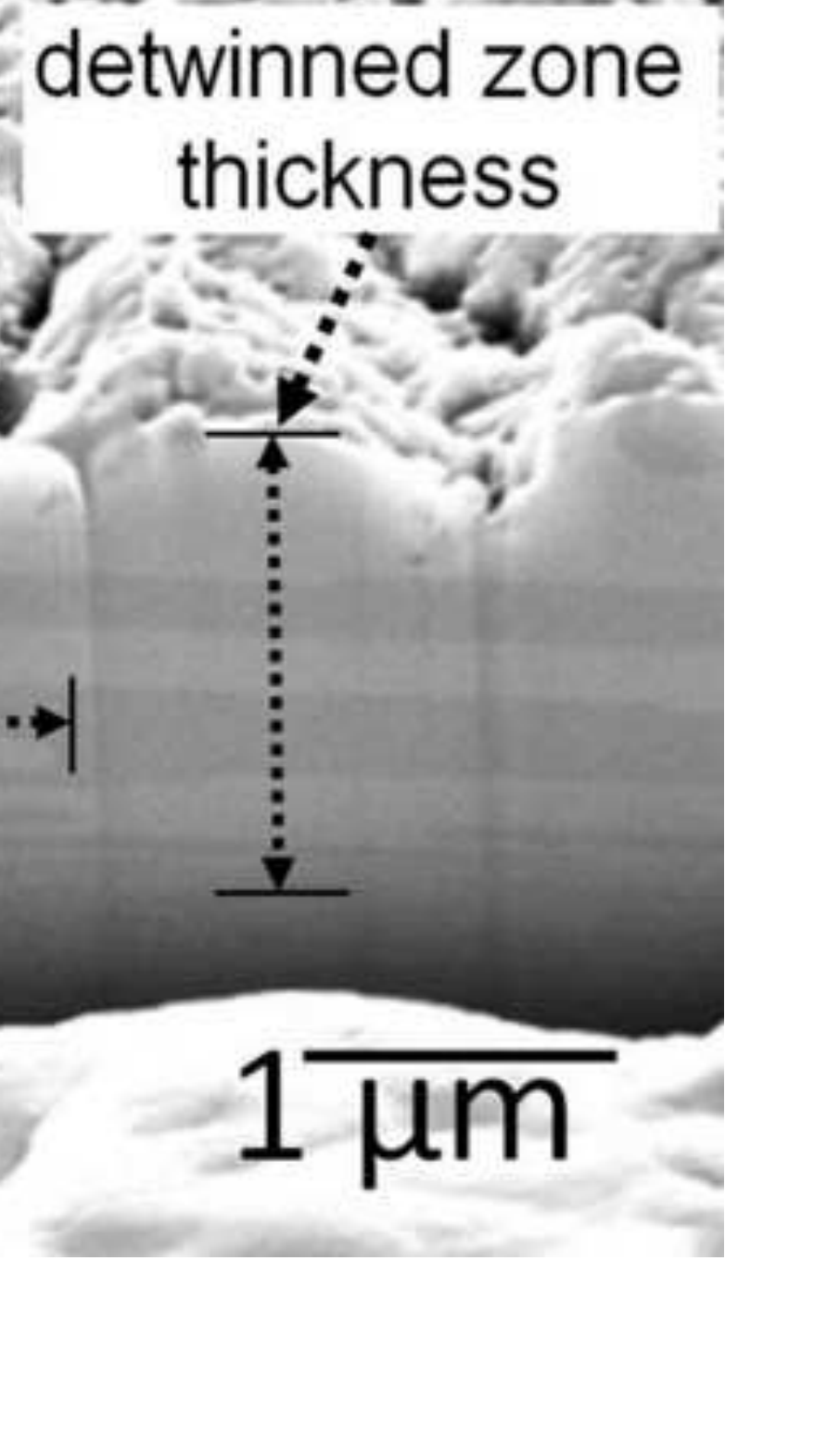

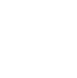

.

.
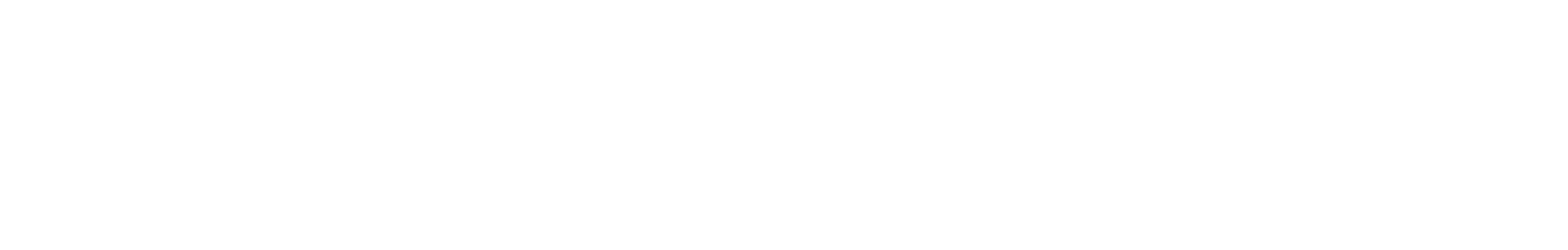


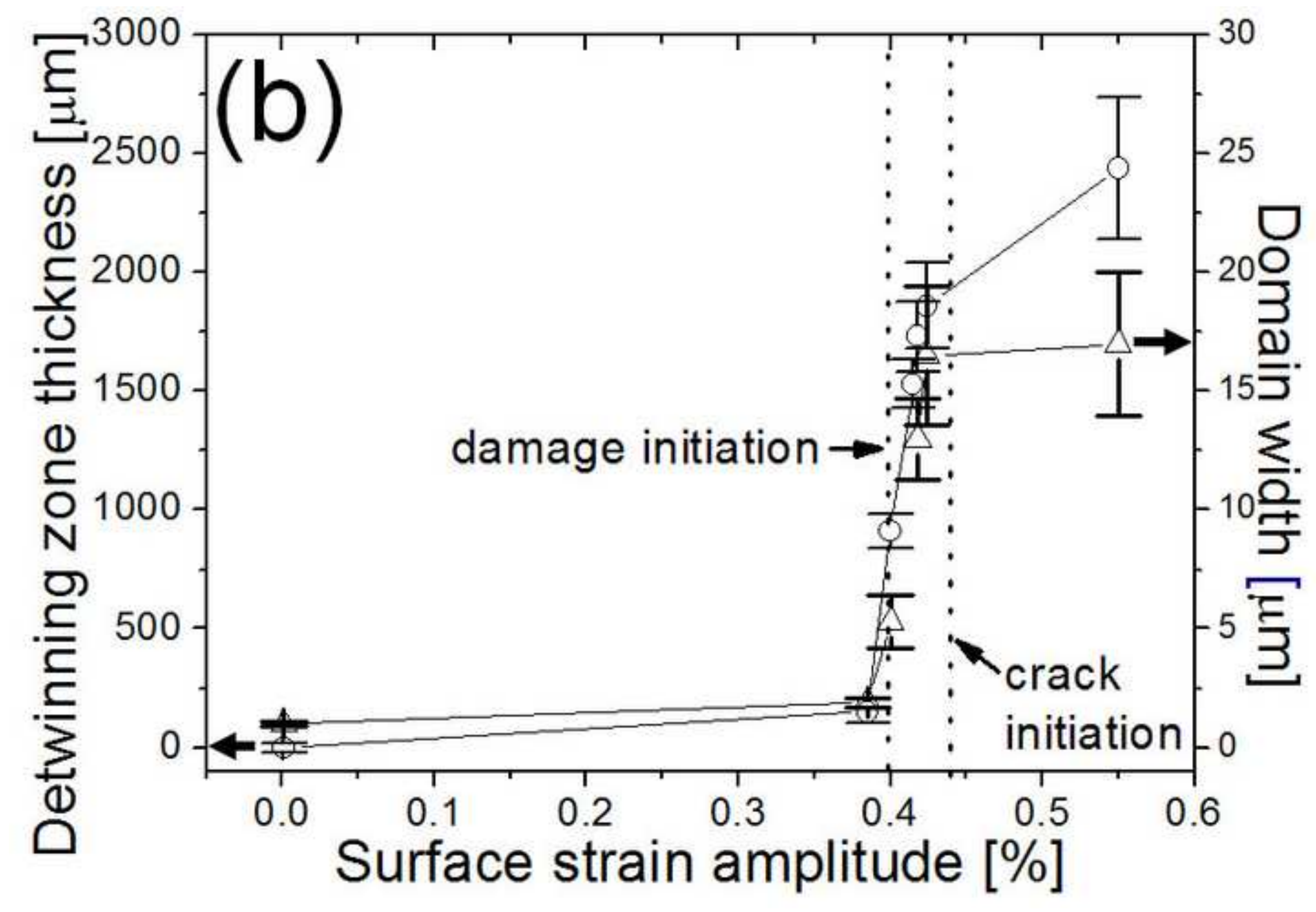




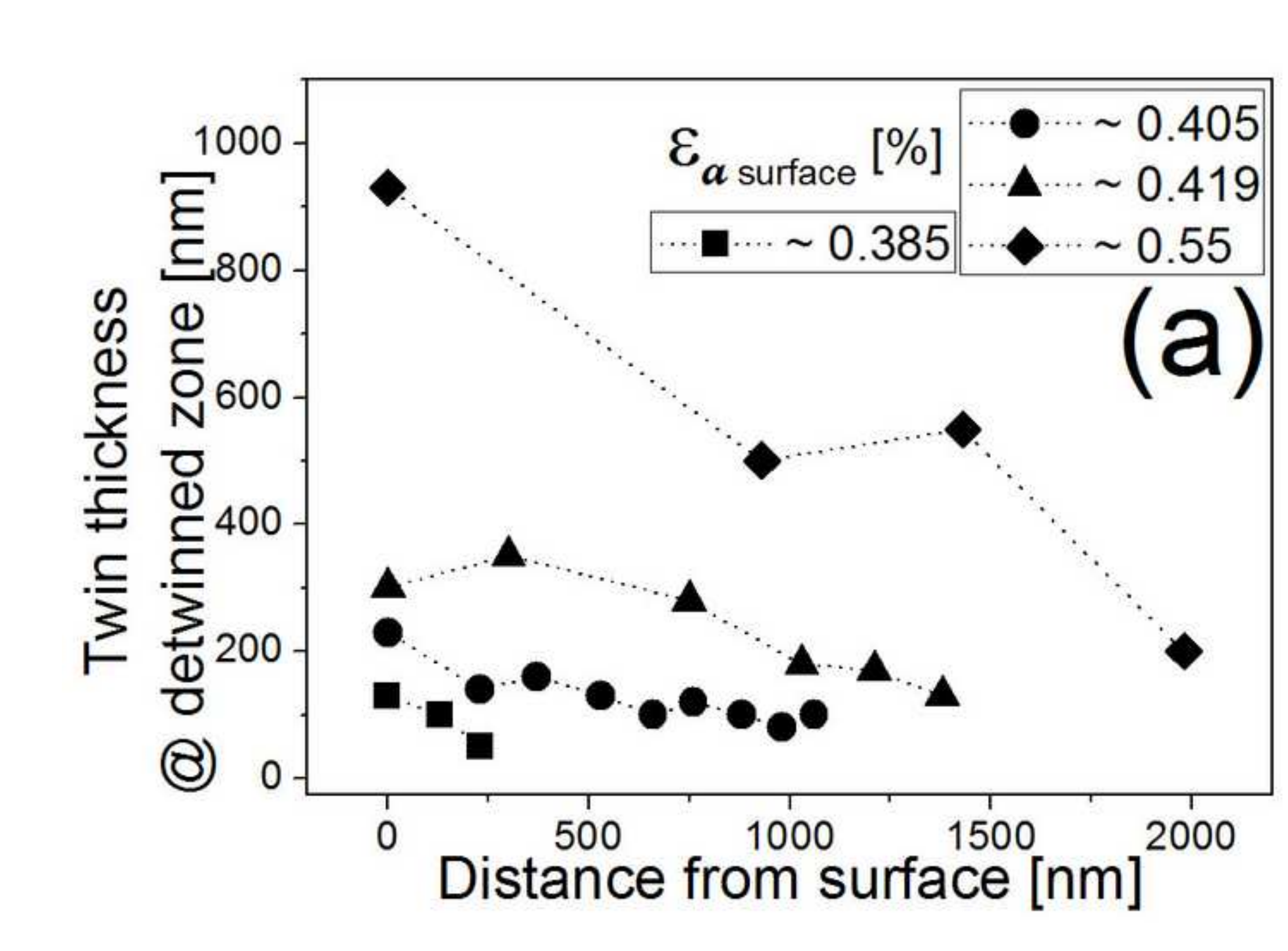

.

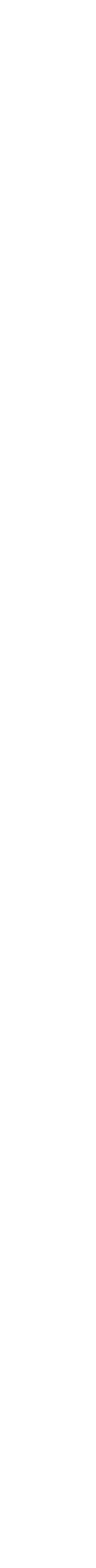

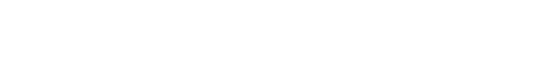

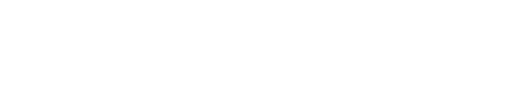




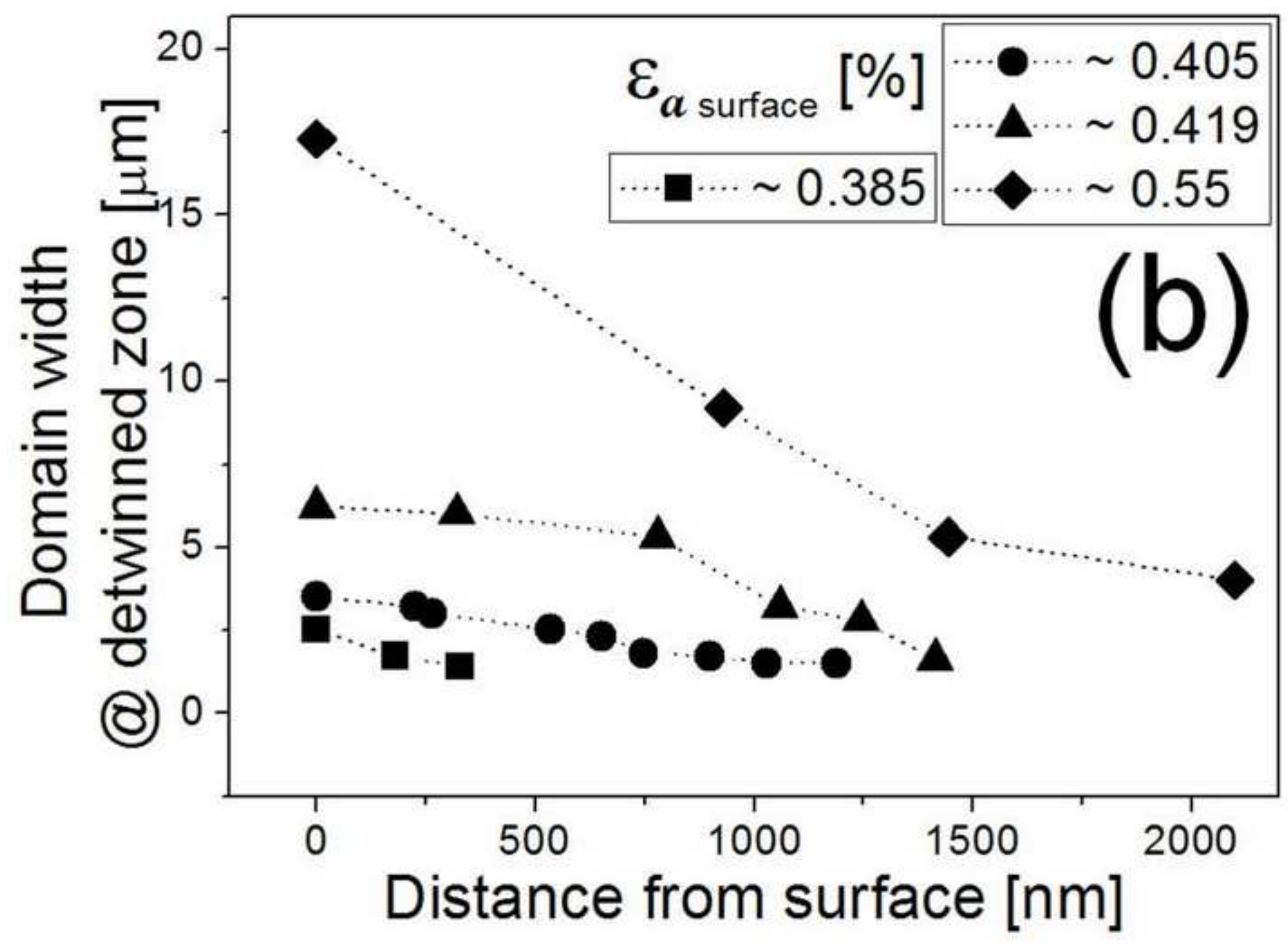




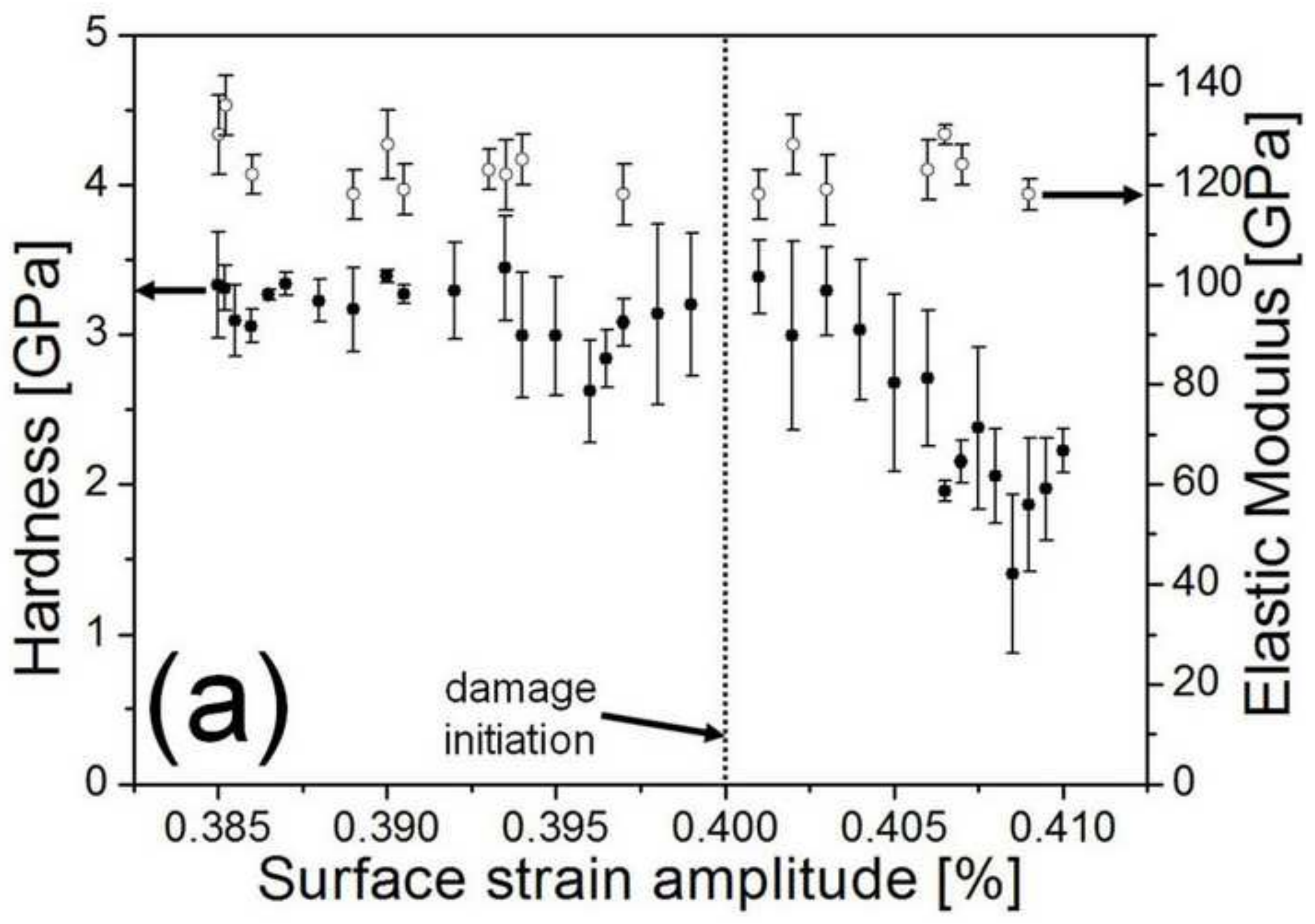




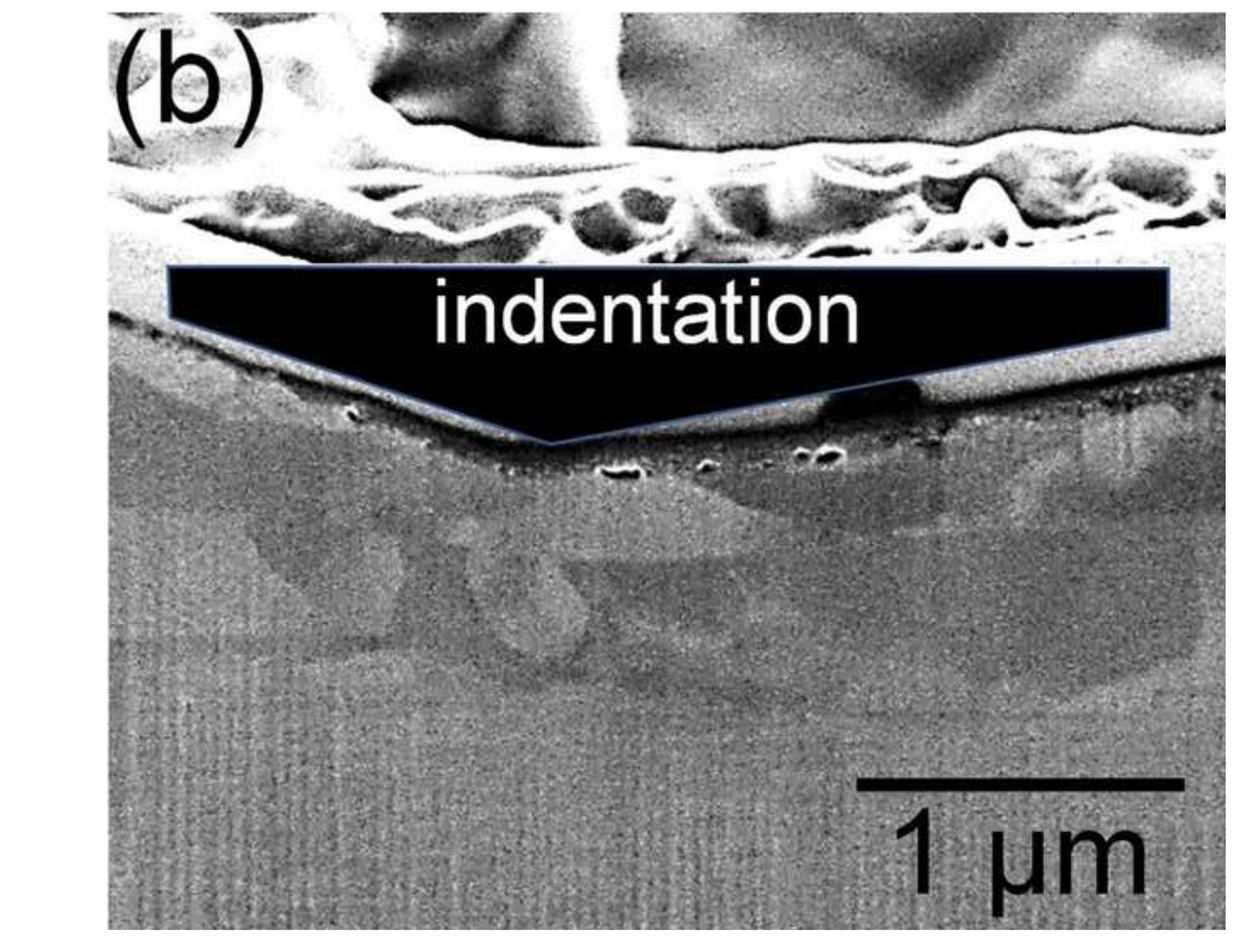

\author{
Firegb
}

-
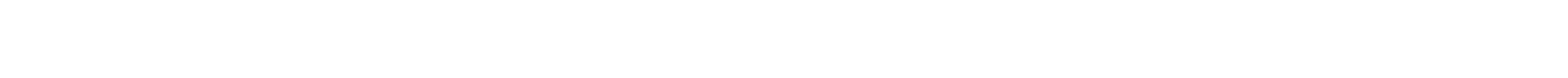

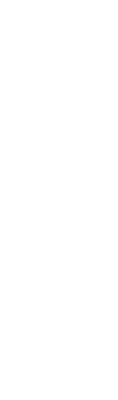
.

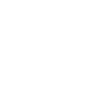

.
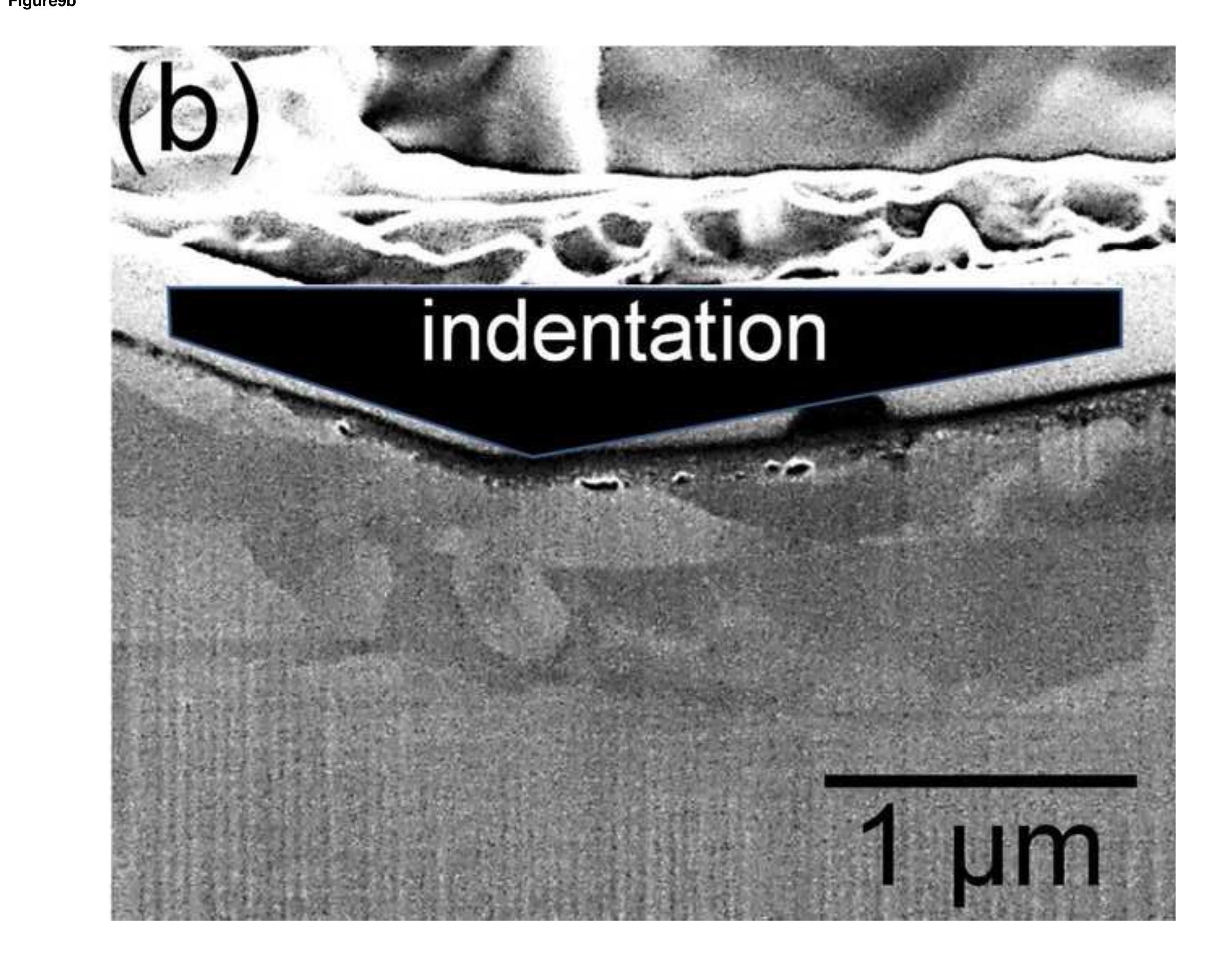


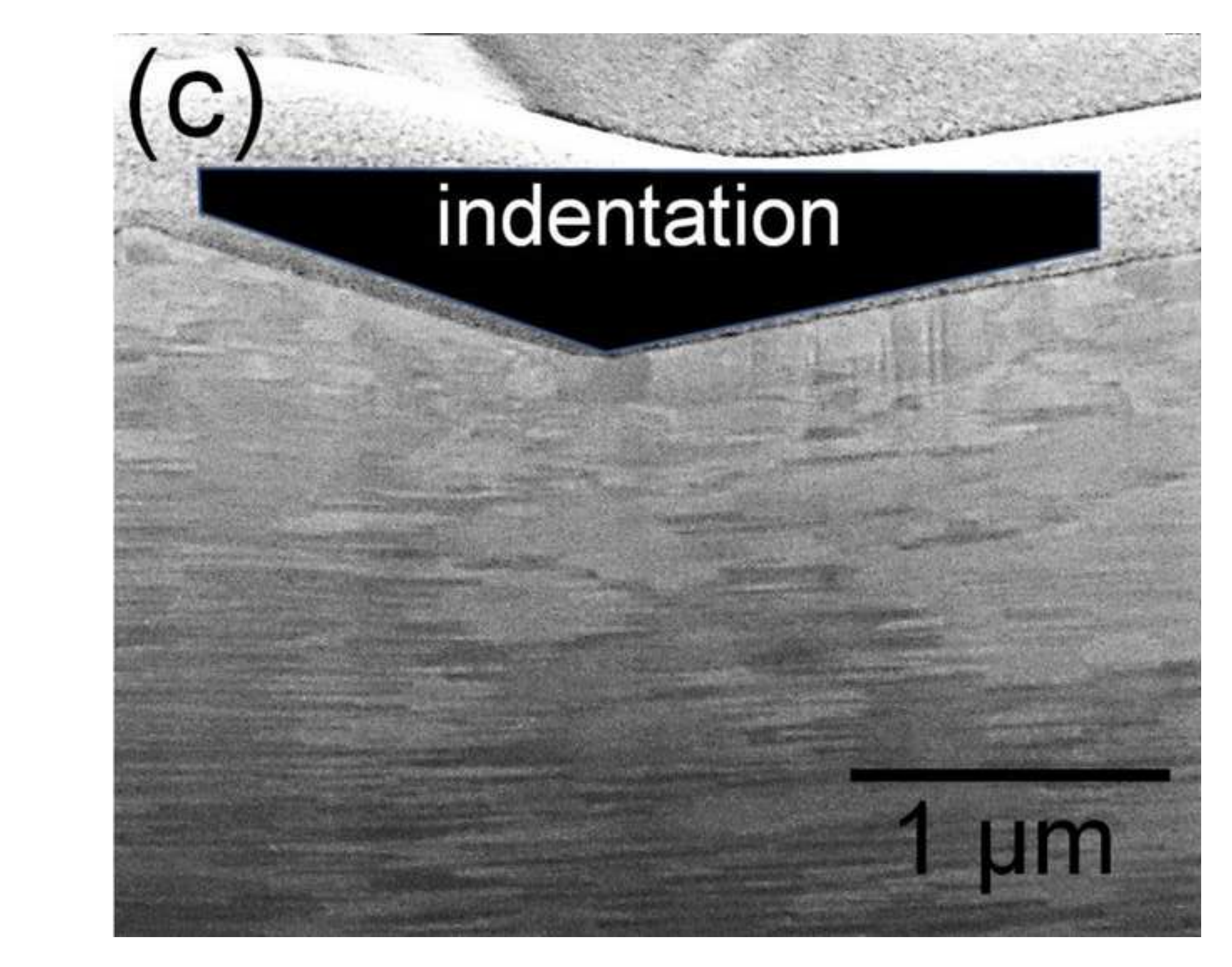

\section{indentation}

\section{.}

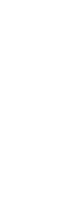

.




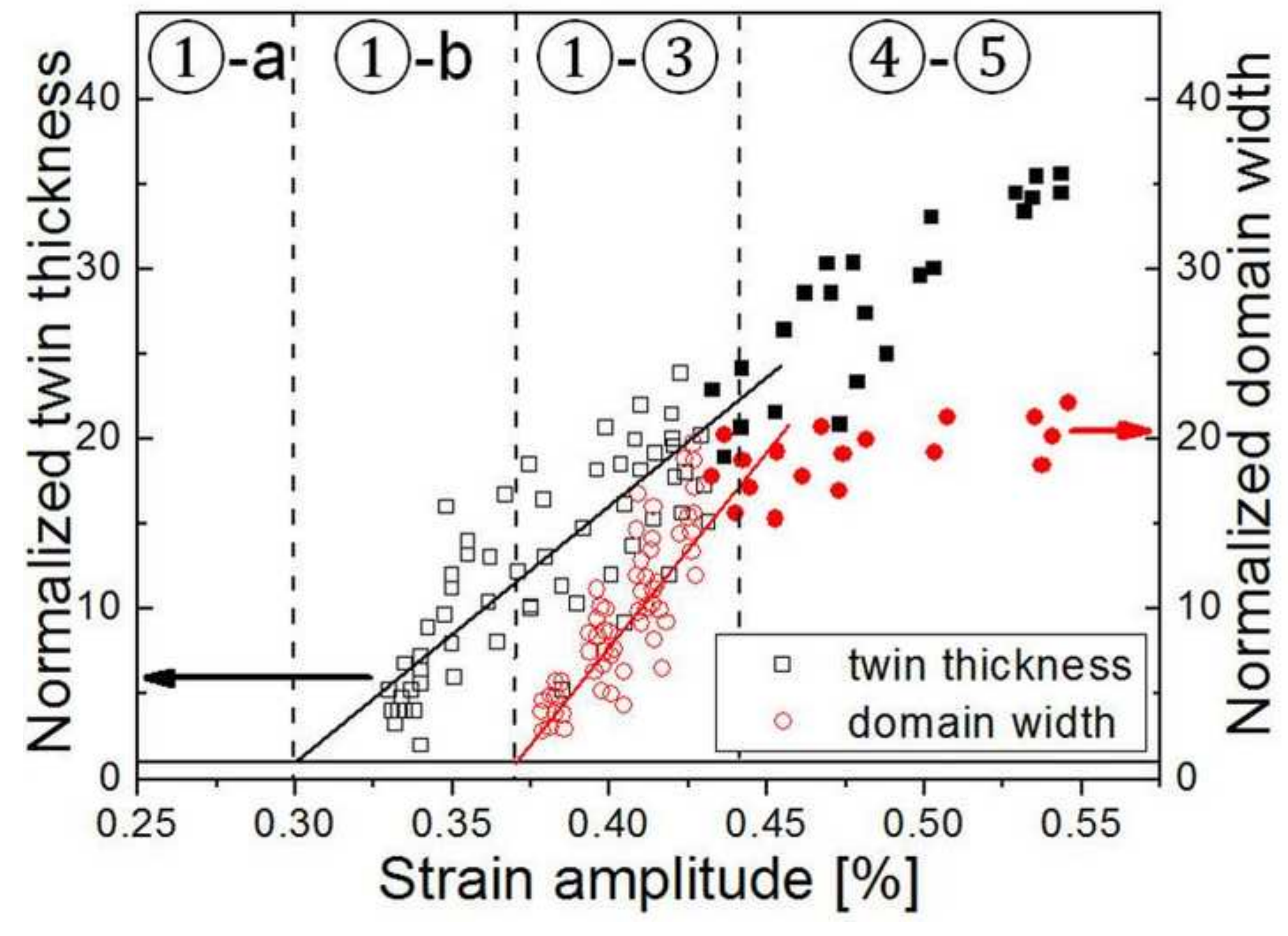

